\title{
Numerical black hole initial data with low eccentricity based on post-Newtonian orbital parameters
}

\author{
Benny Walther, Bernd Brügmann, Doreen Müller \\ Theoretical Physics Institute, University of Jena, 07743 Jena, Germany
}

(Dated: May 5, 2009)

\begin{abstract}
Black hole binaries on non-eccentric orbits form an important subclass of gravitational wave sources, but it is a non-trivial issue to construct numerical initial data with minimal initial eccentricity for numerical simulations. We compute post-Newtonian orbital parameters for quasi-spherical orbits using the method of Buonanno, Chen and Damour (2006) and examine the resulting eccentricity in numerical simulations. Four different methods are studied resulting from the choice of Taylor-expanded or effective-one-body Hamiltonians, and from two choices for the energy flux. For equal-mass, non-spinning binaries the approach succeeds in obtaining low eccentricity numerical initial data with an eccentricity of about $e=0.002$ for rather small initial separations of $D \gtrsim 10 M$. The eccentricity increases for unequal masses and for spinning black holes, but remains smaller than that obtained from previous post-Newtonian approaches. The effective-one-body Hamiltonian offers advantages for decreasing initial separation as expected, but in the context of this study also performs significantly better than the Taylor-expanded Hamiltonian for binaries with spin. For mass ratio 4:1 and vanishing spin, the eccentricity reaches $e=0.004$. For mass ratio 1:1 and aligned spins of size $0.85 M^{2}$ the eccentricity is about $e=0.07$ for the Taylor method and $e=0.014$ for the effective-one-body method.
\end{abstract}

PACS numbers: $\quad 04.25 . \mathrm{D}-$, 04.25.dg, 04.25.Nx

\section{INTRODUCTION}

Gravitational wave astronomy holds the promise to open up an entirely new window into the universe, and a number of ground-based gravitational wave detectors are now collecting data in the quest for first direct detection 1 4. Binary black hole (BBH) mergers are expected to be primary sources of gravitational waves. Theoretical predictions for waves from $\mathrm{BBH}$ mergers are now becoming available for various merger scenarios based on numerical simulations as well as post-Newtonian (PN) approximation methods, with increasing quality and range of validity (e.g. [5, 6]). Among the likely astrophysical scenarios is that of two black holes merging at the end-point of a non-eccentric, quasi-circular inspiral. Although eccentric, non-circular orbits are certainly possible and of interest as well, non-eccentric orbits are expected to be common since the emission of gravitational waves reduces eccentricity on time scales that are short compared to the life time of the binary.

In order to predict waveforms for non-eccentric inspirals, numerical simulations for the Einstein equations have to face the issue of how to construct black hole initial data with minimal initial eccentricity. This is a nontrivial task for several reasons. The main reason is that the initial data have to satisfy the constraint equations of general relativity. Typical initial data constructions allow us to specify the "bare" parameters of a multiple black hole system (masses, positions, momenta, and spins), and the field content (the metric and extrinsic curvature on a hypersurface) is generated by solving elliptic equations. The solution process changes or dresses the bare parameters, e.g. the physical masses and the proper distance between the black holes are obtained as part of the solution. There are therefore two levels of indirectness in the initial data construction. Discrete parameters are translated into fields, and only after solving the constraints the physical parameters are known. The goal is to obtain an accurate approximation to the physical situation at some given instant of time, which as a matter of principle can only be an approximation and cannot be exact because the evolution prior to this time is not available.

With regard to eccentricity, the question is how initial parameters can be found that result in numerical binary inspirals with minimal eccentricity. In fact, most numerical simulations have to contend with a small but sometimes non-negligible amount of eccentricity, and it becomes a quantitative question to what extent the computation of faithful gravitational wave templates is hampered by unwanted eccentricity. For example, the $\mathrm{Nu}-$ merical Injection and Analysis (NINJA) project 7 could benefit from and could evaluate the importance of loweccentricity initial configurations.

Several methods are available to find parameters for approximately circular inspirals. A first approximation is to impose a type of quasi-circularity or quasi-equilibrium condition on the two black holes when solving the constraints, which typically is implemented as an iteration of the orbital parameters and by repeatedly solving the constraints until the quasi-equilibrium condition is satisfied within some given error (see e.g. 8). However, the final judge of the eccentricity contained in initial data is to perform numerical evolutions and to measure the resulting eccentricity. This leads to the immediate suggestion to iterate initial parameters based on the eccen- 
tricity observed in actual evolutions - which, of course, became an option only after numerical simulations of several orbits became possible. Ref. 9] suggests and applies a parameter iteration method based on evolutions with a pseudospectral code. The eccentricity is estimated after one or two orbits, and the run is restarted with improved parameters. The appeal of this method is its generality. It still has to be determined how well it can work with less accurate finite differencing codes. In particular, due to artificial waves and gauge issues it is not clear how many orbits are required to measure the eccentricity with sufficient accuracy in the numerical simulation. For short simulations of only a few orbits, several iterations of the method may be computationally expensive.

Another natural idea in this context is to obtain orbital parameters from PN methods. Once PN orbital parameters for minimal eccentricity within the PN approximation are found, they can be used as input for a constraint solution scheme within the full theory. Even simple implementations of this method can work surprisingly well. For example in [10, (see also [1, 12]), it turns out that already a simple 3PN formula to compute the tangential momentum $P_{t}$ for a PN circular orbit with vanishing radial momentum $P_{R}$, leads to approximately circular inspiral initial data when combined with the puncture method [13] to construct binary black hole initial data.

Typical numerical simulations start shortly before the merger, say at an initial separation that allows 5 to 15 orbits before merger. In this regime, the PN approach becomes increasingly inaccurate with decreasing separation as the dynamic, strong field effects of the inspiral become severe. In order to improve on the PN parameters of [10], we can look to more sophisticated PN approximations, say involving the effective-one-body (EOB) approach [14 17. In particular, the PN method should also supply a non-vanishing radial momentum $P_{R}$. Ironically, it is the use of "quasi-circular" initial parameters with vanishing radial momentum that leads to non-circular, eccentric inspirals, since by definition a spiral requires a non-vanishing radial momentum.

We note in passing that there also exists a method to translate PN data including not only parameters $P_{t}$ and $P_{R}$, but also the PN field content to numerical initial data [18. The result is black hole puncture data with more realistic gravitational wave content [19], a feature shared with the periodic standing wave approximation, e.g. 20]. This feature is not essential for numerical evolutions, but in any case is not implemented by the methods discussed here.

One way to obtain improved PN parameters for inspirals with small eccentricity is to start with a simple initial guess and to perform evolutions of a few hundred orbits in the PN Hamiltonian formulation until the orbit has circularized to a high degree due to the emission of gravitational waves 21]. In terms of computational effort, note that once the PN formulas for the Hamiltonian system have been coded, the time integration is easily handled by a standard Mathematica function in less than a minute. The elegance of the PN evolution method is that for any reasonable initial guess the PN evolution leads to a circularized inspiralling orbit due to the physical process of wave emission, and this is achieved with little additional effort.

For numerical simulations of equal mass binaries without spin, the residual eccentricity is reduced by up to a factor of five by the PN evolution method 21] compared to simple 2PN quasi-circular parameters [22, 23, with an eccentricity $e<0.002$ at an initial separation of $D=11 M$. For comparison, several equal mass data sets are mentioned in 24], with $e=0.0016$ the lowest value used for finite differencing codes, $e=0.008$ a typical value prior to the recent improvements, but $e<5 \times 10^{-5}$ for the iteration method in a pseudospectral code 9 . For more general binaries with spin and unequal masses, there is some improvement of a typical initial guess using the PN evolution method, but the numerical eccentricity is not always as small as one may wish [25, 26]. As suggested in [27], it may be possible to add one iteration step to improve the situation for binaries with spin.

The PN evolution method also demonstrates how large the eccentricity can be within the PN method itself if certain simple prescriptions for quasi-circular orbit parameters are used. On the other hand, the question arises how well a more sophisticated PN method can achieve orbital parameters for non-eccentric inspirals. In particular, we want to ask the question whether there is a PN method that produces orbital parameters directly (without resorting to PN evolutions) that leads to reduced eccentricity for numerical simulations. As explained above, we do not look for a method that reduces the eccentricity to zero, but any PN parameter proposal can be called successful e.g. if it reduces the eccentricity below the level of changes introduced by the constraint solving process. In any case, PN parameters can provide a good initial guess for an iteration based on full numerical simulations.

One of the problems associated with the application of PN methods is that there is not one, but a multitude of approaches that differ in strategy as well as in small but important details. For example, two sets of PN initial parameters may be equivalent up to a certain PN order, but show differences in numerical simulations, which may be sensitive to the higher order terms that have been neglected.

In this paper we focus on the proposal of Buonanno, Chen, and Damour 28] to compute quasi-spherical PN initial data. This work was based on the Hamiltonian formulation plus the known energy-loss rate and considered an adiabatic sequence of spherical orbits. That means the tangential momentum is determined by demanding a constant binary separation when using the conservative part only. In a second step the radial component is derived by asking for the rate at which these spherical orbits are (adiabatically) traversed by including the energy flux. The approach is capable of incorporating spin-orbit interactions to leading order, which may present a simple way of improving initial data for spinning binaries. 
The present paper implements the suggestions and the algorithm of [28] and modifies it to be more directly applicable to dynamical variables instead of kinematical ones, i.e. ADMTT momenta instead of velocities. Following 28] we implement two variants of PN Hamiltonians, the usual Taylor-expanded and the EOB version. We also consider two versions of the energy flux, the classical Taylor-expanded flux for circularly orbiting bodies and a particular Padé approximant of it. When combined with the two choices of Hamiltonians this results in four different versions to study.

In Section II we provide a detailed survey of the required equations. We further examine the transition from EOB coordinates to ADMTT-type coordinates, which becomes necessary when extracting initial data from the EOB Hamiltonian. The initial data algorithm itself is described in Section [II] Section [IV is dedicated to the exploration of these data in numerical simulations with the BAM code. By measuring the orbital eccentricity obtained in numerical simulations we can argue which of the four data types is most appropriate. The investigation includes mass ratios 1:1, 2:1, and 4:1 for vanishing spin, several cases of spin aligned to the orbital angular momentum for mass ratio 1:1, one anti-aligned case and one situation with arbitrarily chosen spins. Especially for binaries with spin the resulting eccentries are found to be quite large, but initial data involving the EOB Hamiltonian still give reasonably small eccentricity.

\section{POST-NEWTONIAN EQUATIONS OF MOTION FOR SPINNING BINARY SYSTEMS}

This section provides the required equations of motion of a black-hole binary system consisting of objects of masses $m_{a}$, positions $\boldsymbol{X}_{a}$, momenta $\boldsymbol{P}_{a}$ and spins $\boldsymbol{S}_{a}$ $(a=1,2)$. For our purposes it is sufficient to restrict considerations to the center-of-mass dynamics, where $\boldsymbol{P} \equiv \boldsymbol{P}_{1}=-\boldsymbol{P}_{2}$. Following [28, we use a Hamiltonian description in either ADMTT gauge or in EOB coordinates.

The Hamiltonian formalism turns out to be very useful when working with the canonically conjugate position and momentum variables and in distinguishing conservative and radiative effects. The system's equations of motion take the form

$$
\begin{aligned}
\frac{\mathrm{d} \boldsymbol{X}}{\mathrm{d} t} & =\{\boldsymbol{X}, H\}=\frac{\partial H}{\partial \boldsymbol{P}}, \\
\frac{\mathrm{d} \boldsymbol{P}}{\mathrm{d} t} & =\{\boldsymbol{P}, H\}+\boldsymbol{F}=-\frac{\partial H}{\partial \boldsymbol{X}}+\boldsymbol{F}, \\
\frac{\mathrm{d} \boldsymbol{S}_{a}}{\mathrm{~d} t} & =\left\{\boldsymbol{S}_{a}, H\right\}=\frac{\partial H}{\partial \boldsymbol{S}_{a}} \times \boldsymbol{S}_{a},
\end{aligned}
$$

where $\boldsymbol{F}$ labels the non-conservative force and $\times$ denotes the usual vector cross product. The conservative part consists of an orbital and a spin contribution and we will use the total Hamiltonian formed by the sum of three components,

$$
\begin{aligned}
H\left(\boldsymbol{X}, \boldsymbol{P}, \boldsymbol{S}_{1}, \boldsymbol{S}_{2}\right)= & H^{0}(\boldsymbol{X}, \boldsymbol{P})+H_{\mathrm{SO}}\left(\boldsymbol{X}, \boldsymbol{P}, \boldsymbol{S}_{1}, \boldsymbol{S}_{2}\right) \\
& +H_{\mathrm{SS}}\left(\boldsymbol{X}, \boldsymbol{P}, \boldsymbol{S}_{1}, \boldsymbol{S}_{2}\right)
\end{aligned}
$$

The individual terms are presented in the subsequent paragraphs.

\section{A. Orbital Hamiltonian}

We consider two versions of the orbital contribution $H^{0}$, the standard $3 \mathrm{PN}$ accurate Taylor-expanded Hamiltonian (TH) derived by Damour, Jaranowski, and Schäfer [16, 29 31, and the effective-one-body Hamiltonian (EH) given by Buonanno, Damour, Jaranowski, and Schäfer at 3PN order in [15, 16] (given first at $2 \mathrm{PN}$ in [14). It is crucial to note that the two Hamiltonians are defined for different coordinate systems. The Taylor-expanded Hamiltonian uses ADMTT-type coordinates denoted by $\boldsymbol{X}, \boldsymbol{P}$, where, however, a small deviation from the actual ADMTT gauge arises at the 3PN-level [29]. The EOB coordinates, indicated by primes $\boldsymbol{X}^{\prime}, \boldsymbol{P}^{\prime}$, are related to the ADMTT coordinates by a canonical transformation. The character of this transformation will be investigated in Section II D below. Some equations are valid for both approaches, TH and $\mathrm{EH}$, and in these cases the primes are omitted as in Eqns. (1)- 4).

Both versions of the Hamiltonian will be given explicitly. By introducing reduced coordinates $\boldsymbol{q}=$ $\boldsymbol{X} /(G M)=\left(\boldsymbol{X}_{1}-\boldsymbol{X}_{2}\right) /(G M)$ and $\boldsymbol{p}=\boldsymbol{P} / \mu$, where $M=m_{1}+m_{2}$ and $\mu=m_{1} m_{2} / M$, the Taylor-expanded version reads 


$$
H_{\text {Taylor }}^{0}(\boldsymbol{q}, \boldsymbol{p})=M c^{2}+\mu\left[\hat{H}_{\mathrm{N}}(\boldsymbol{q}, \boldsymbol{p})+\frac{1}{c^{2}} \hat{H}_{1 \mathrm{PN}}(\boldsymbol{q}, \boldsymbol{p})+\frac{1}{c^{4}} \hat{H}_{2 \mathrm{PN}}(\boldsymbol{q}, \boldsymbol{p})+\frac{1}{c^{6}} \hat{H}_{3 \mathrm{PN}}(\boldsymbol{q}, \boldsymbol{p})\right]
$$

with

$$
\begin{aligned}
& \hat{H}_{\mathrm{N}}(\boldsymbol{q}, \boldsymbol{p})=\frac{\boldsymbol{p}^{2}}{2}-\frac{1}{q}, \\
& \hat{H}_{1 \mathrm{PN}}(\boldsymbol{q}, \boldsymbol{p})=\frac{1}{8}(3 \nu-1)\left(\boldsymbol{p}^{2}\right)^{2}-\frac{1}{2}\left[(3+\nu) \boldsymbol{p}^{2}+\nu(\boldsymbol{n} \boldsymbol{p})^{2}\right] \frac{1}{q}+\frac{1}{2 q^{2}}, \\
& \hat{H}_{2 \mathrm{PN}}(\boldsymbol{q}, \boldsymbol{p})=\frac{1}{16}\left(1-5 \nu+5 \nu^{2}\right)\left(\boldsymbol{p}^{2}\right)^{3}+\frac{1}{8}\left[\left(5-20 \nu-3 \nu^{2}\right)\left(\boldsymbol{p}^{2}\right)^{2}-2 \nu^{2}(\boldsymbol{n} \boldsymbol{p})^{2} \boldsymbol{p}^{2}-3 \nu^{2}(\boldsymbol{n} \boldsymbol{p})^{4}\right] \frac{1}{q} \\
&+\frac{1}{2}\left[3 \nu(\boldsymbol{n} \boldsymbol{p})^{2}+(5+8 \nu) \boldsymbol{p}^{2}\right] \frac{1}{q^{2}}-\frac{1}{4}(1+3 \nu) \frac{1}{q^{3}}, \\
& \hat{H}_{3 \mathrm{PN}}(\boldsymbol{q}, \boldsymbol{p})= \frac{1}{128}\left(-5+35 \nu-70 \nu^{2}+35 \nu^{3}\right)\left(\boldsymbol{p}^{2}\right)^{4}+\frac{1}{16}\left[\left(-7+42 \nu-53 \nu^{2}-5 \nu^{3}\right)\left(\boldsymbol{p}^{2}\right)^{3}\right. \\
&+\left.(2-3 \nu) \nu^{2}(\boldsymbol{n} \boldsymbol{p})^{2}\left(\boldsymbol{p}^{2}\right)^{2}+3(1-\nu) \nu^{2}(\boldsymbol{n} \boldsymbol{p})^{4} \boldsymbol{p}^{2}-5 \nu^{3}(\boldsymbol{n} \boldsymbol{p})^{6}\right] \frac{1}{q} \\
&+ {\left[\frac{1}{16}\left(-27+136 \nu+109 \nu^{2}\right)\left(\boldsymbol{p}^{2}\right)^{2}+\frac{1}{16}(17+30 \nu) \nu(\boldsymbol{n} \boldsymbol{p})^{2} \boldsymbol{p}^{2}+\frac{1}{12}(5+43 \nu) \nu(\boldsymbol{n} \boldsymbol{p})^{4}\right] \frac{1}{q^{2}} } \\
&+\left\{\left[-\frac{25}{8}+\left(\frac{1}{64} \pi^{2}-\frac{335}{48}\right) \nu-\frac{23}{8} \nu^{2}\right] \boldsymbol{p}^{2}+\left(-\frac{85}{16}-\frac{3}{64} \pi^{2}-\frac{7}{4} \nu\right) \nu(\boldsymbol{n p})^{2}\right\} \frac{1}{q^{3}} \\
&+ {\left[\frac{1}{8}+\left(\frac{109}{12}-\frac{21}{32} \pi^{2}\right) \nu\right] \frac{1}{q^{4}}, }
\end{aligned}
$$

where additionally $q=|\boldsymbol{q}|, \boldsymbol{n}=\boldsymbol{q} / q$ and $\nu=\mu / M$ have been used. We already plugged in the appropriate values of the ambiguity parameters $\omega_{\text {static }}$ and $\omega_{\text {kinetic }}$.

The EOB Hamiltonian is given by the expression

$$
H_{\mathrm{EOB}}^{0}\left(\boldsymbol{q}^{\prime}, \boldsymbol{p}^{\prime}\right)=M c^{2} \sqrt{1+2 \nu\left(\frac{H_{\mathrm{eff}}\left(\boldsymbol{q}^{\prime}, \boldsymbol{p}^{\prime}\right)-\mu c^{2}}{\mu c^{2}}\right)},
$$

with

$$
H_{\mathrm{eff}}\left(\boldsymbol{q}^{\prime}, \boldsymbol{p}^{\prime}\right)=\mu c^{2} \sqrt{A\left(q^{\prime}\right)\left[1+\frac{\boldsymbol{p}^{\prime 2}}{c^{2}}+\left(\frac{A\left(q^{\prime}\right)}{D\left(q^{\prime}\right)}-1\right) \frac{\left(\boldsymbol{n}^{\prime} \cdot \boldsymbol{p}^{\prime}\right)^{2}}{c^{2}}+\frac{1}{c^{4}} \frac{1}{q^{\prime 2}}\left(z_{1}\left(\boldsymbol{p}^{\prime 2}\right)^{2}+z_{2} \boldsymbol{p}^{\prime 2}\left(\boldsymbol{n}^{\prime} \cdot \boldsymbol{p}^{\prime}\right)^{2}+z_{3}\left(\boldsymbol{n}^{\prime} \cdot \boldsymbol{p}^{\prime}\right)^{4}\right)\right]}
$$

where $A\left(\boldsymbol{q}^{\prime}\right)$ is the Padé-resummed function

$$
A\left(q^{\prime}\right)=\frac{q^{\prime 3}(8-2 \nu)+\frac{1}{c^{2}} q^{\prime 2}\left(a_{4}+8 \nu-16\right)}{q^{\prime 3}(8-2 \nu)+\frac{1}{c^{2}} q^{\prime 2}\left(a_{4}+4 \nu\right)+\frac{1}{c^{4}} q^{\prime}\left(2 a_{4}+8 \nu\right)+\frac{1}{c^{6}} 4\left(a_{4}+\nu^{2}\right)}
$$

and the remaining quantities are

$$
\begin{aligned}
a_{4} & =\left[\left(\frac{94}{3}-\frac{41}{32} \pi^{2}\right)-\frac{z_{1}}{\nu}\right] \nu, \\
D\left(q^{\prime}\right) & =1-\frac{1}{c^{4}} \frac{6 \nu}{q^{\prime 2}}+\frac{1}{c^{6}}\left[7 \frac{z_{1}}{\nu}+\frac{z_{2}}{\nu}+(3 \nu-26)\right] \frac{\nu}{q^{\prime 3}}, \\
z_{1} & =z_{2}=0 \quad \text { and } \quad z_{3}=2(4-3 \nu) \nu .
\end{aligned}
$$

As before we make use of reduced variables in the formulation of the EOB Hamiltonian. 


\section{B. Spin Hamiltonian}

The spin part will be considered to leading order only. These terms can be separated into spin-orbit and spinspin interactions and have been known for a long time, see for example [28, 32. We have

$$
\begin{aligned}
H_{\mathrm{SO}} & =2 \frac{G}{c^{2}} \frac{\boldsymbol{S}_{\mathrm{eff}} \cdot \boldsymbol{L}}{R^{3}} \\
\boldsymbol{S}_{\mathrm{eff}} & =\left(1+\frac{3}{4} \frac{m_{2}}{m_{1}}\right) \boldsymbol{S}_{1}+\left(1+\frac{3}{4} \frac{m_{1}}{m_{2}}\right) \boldsymbol{S}_{2} \\
H_{\mathrm{SS}} & =H_{S_{1} S_{1}}+H_{S_{1} S_{2}}+H_{S_{2} S_{2}} \\
H_{S_{1} S_{2}} & =\frac{G}{c^{2}} \frac{1}{R^{3}}\left[3\left(\boldsymbol{S}_{1} \cdot \boldsymbol{N}\right)\left(\boldsymbol{S}_{2} \cdot \boldsymbol{N}\right)-\left(\boldsymbol{S}_{1} \cdot \boldsymbol{S}_{2}\right)\right] \\
H_{S_{1} S_{1}} & =\frac{G}{c^{2}} \frac{1}{2 R^{3}}\left[3\left(\boldsymbol{S}_{1} \cdot \boldsymbol{N}\right)\left(\boldsymbol{S}_{1} \cdot \boldsymbol{N}\right)-\left(\boldsymbol{S}_{1} \cdot \boldsymbol{S}_{1}\right)\right] \frac{m_{2}}{m_{1}}, \\
H_{S_{2} S_{2}} & =1 \rightleftharpoons 2 .
\end{aligned}
$$

Here, $\boldsymbol{L}=\boldsymbol{X} \times \boldsymbol{P}$ is the orbital angular momentum and $\boldsymbol{N}$ is the same unit vector as $\boldsymbol{n}$ above. We assume the Newton-Wigner spin supplementary condition, which provides the notion of the spin vector and affects the definition of the bodies' worldlines.

In what follows Eqns. (16)- 20 are presumed to hold for ADMTT as well as EOB coordinates. This, however, is an approximation as already noted in [28]. There exists a more sophisticated and potentially more accurate way of including spin effects in the EOB framework [17, but the Hamiltonian associated with this approach contains spin-orbit and spin-spin effects mixed in a complicated way, and it seems that these terms cannot be separated in a consistent manner. The initial-data algorithm we present, however, relies on neglecting the spin-spin terms for the sake of obtaining spherical orbits. Hence, in the EOB case we follow [28] by just employing the same simple, additive spin Hamiltonian as in the ADM framework. We discuss the consequences of this approximation below when analyzing the canonical transformation between the two coordinate systems.

\section{Radiation-reaction force}

The radiation-reaction-force term $\boldsymbol{F}$ to be applied in Eq. (2) is the expression derived by 28]. It has the feature that it is formulated in terms of dynamical quantities $(\boldsymbol{X}, \boldsymbol{P})$ instead of kinematical ones $(\boldsymbol{X}, \dot{\boldsymbol{X}})$. It also includes spin interactions to linear order and reads

$$
\begin{aligned}
\boldsymbol{F}= & \frac{1}{\omega|\boldsymbol{L}|} \frac{\mathrm{d} E}{\mathrm{~d} t} \boldsymbol{P}+\frac{8}{15} \nu^{2} \frac{v_{\omega}^{8}}{\boldsymbol{L}^{2} R}\left\{\left(61+48 \frac{m_{2}}{m_{1}}\right) \boldsymbol{P} \cdot \boldsymbol{S}_{1}\right. \\
& \left.+\left(61+48 \frac{m_{1}}{m_{2}}\right) \boldsymbol{P} \cdot \boldsymbol{S}_{2}\right\} \boldsymbol{L}
\end{aligned}
$$

The invariant velocity parameter

$$
v_{\omega}=\left(\frac{G M \omega}{c^{3}}\right)^{1 / 3}
$$

based on the orbital frequency $\omega=\dot{\varphi}$ has been introduced, although this brings the kinematical quantities back into play.

Eq. 22 is valid for (quasi-) circular orbits only, which is a result of simplifications in its derivation and due to the fact that the quantity $\mathrm{d} E / \mathrm{d} t$ is the well-known energy-flux function of circularly orbiting masses [28, 3337. In particular, its standard form (up to 3.5PN order) does not account for any eccentricity parameter,

$$
\begin{aligned}
\frac{\mathrm{d} E}{\mathrm{~d} t}= & -\frac{32}{5} \nu^{2} v_{\omega}^{10}\left\{1+f_{2}(\nu) v_{\omega}^{2}+\left[f_{3}(\nu)+f_{3 \mathrm{SO}}\right] v_{\omega}^{3}\right. \\
& +\left[f_{4}(\nu)+f_{4 \mathrm{SS}}\right] v_{\omega}^{4}+f_{5}(\nu) v_{\omega}^{5}+f_{6}(\nu) v_{\omega}^{6} \\
& \left.+f_{l 6} v_{\omega}^{6} \ln \left(4 v_{\omega}\right)+f_{7}(\nu) v_{\omega}^{7}\right\} .
\end{aligned}
$$

These equations are also available for non-vanishing eccentricity in 38. The expansion coefficients read

$$
\begin{aligned}
& f_{2}(\nu)=-\frac{1247}{336}-\frac{35}{12} \nu, \\
& f_{3}(\nu)=4 \pi, \\
& f_{4}(\nu)=-\frac{44711}{9072}+\frac{9271}{504} \nu+\frac{65}{18} \nu^{2}, \\
& f_{5}(\nu)=-\left(\frac{8191}{672}+\frac{583}{24} \nu\right) \pi, \\
& f_{6}(\nu)=\frac{6643739519}{69854400}+\frac{16}{3} \pi^{2}-\frac{1712}{105} \gamma_{\mathrm{E}} \\
& +\left(-\frac{134543}{7776}+\frac{41}{48} \pi^{2}\right) \nu-\frac{94403}{3024} \nu^{2}-\frac{775}{324} \nu^{3}, \\
& f_{l 6}=-\frac{1712}{105} \\
& f_{7}(\nu)=\left(-\frac{16285}{504}+\frac{214745}{1728} \nu+\frac{193385}{3024} \nu^{2}\right) \pi, \\
& f_{3 \mathrm{SO}}=-\left(\frac{11}{4}+\frac{5}{4} \frac{m_{2}}{m_{1}}\right) \frac{\hat{\boldsymbol{L}} \cdot \boldsymbol{S}_{1}}{M^{2}}-\left(\frac{11}{4}+\frac{5}{4} \frac{m_{1}}{m_{2}}\right) \frac{\hat{\boldsymbol{L}} \cdot \boldsymbol{S}_{2}}{M^{2}} \\
& f_{4 \mathrm{SS}}=\frac{\nu}{48 m_{1}^{2} m_{2}^{2}}\left[289\left(\hat{\boldsymbol{L}} \cdot \boldsymbol{S}_{1}\right)\left(\hat{\boldsymbol{L}} \cdot \boldsymbol{S}_{2}\right)-103\left(\boldsymbol{S}_{1} \cdot \boldsymbol{S}_{2}\right)\right] \\
& +\mathcal{O}\left(S_{1}^{2}\right)+\mathcal{O}\left(S_{2}^{2}\right)
\end{aligned}
$$

where $\gamma_{\mathrm{E}}=0.577215 \ldots$ is Euler's gamma and $\hat{\boldsymbol{L}}$ gives the unit vector in the direction of $\boldsymbol{L}$. Note that the term 
$f_{4 \mathrm{SS}}$ is not fully known yet, but we will not be using it anyway.

The force expression 22 actually does not enter the initial data algorithm presented in Section III below, however the energy flux (24) does. The fact that the flux is unable to describe eccentricity damping properly is not supposed to affect the algorithm. This is because we will merely ask for the shrinkage rate of circular (resp. spherical) orbits at one particular radius. In contrast, the application of the flux and the force $\boldsymbol{F}$ in PN simulations with noticeable (e.g. spin induced) eccentricity might give inaccurate results. In such cases we may want to consider time dependent flux expressions or time averages over elliptical orbits [33].

Concerning the PN convergence of the flux function, several authors have shown that the Padé-resummation technique is capable of improving convergence, in particular in the test-mass limit. A recent paper, however, claims that no advantages can be found using the Padéresummation approach for several specific examples [39]. In order to examine this issue in the context of our eccentricity study, we implement the standard flux (24), but also consider a direct P-approximant of the flux. The final expression is too lengthy to be shown here. Since computer-algebra software provides an efficient way to perform the resummation, we restrict ourselves to a brief summary of the required procedure. A more detailed description can be found in 40, 41.

Padé's method defines the resummation of a plain Taylor series. Referring to (24), we first separate the spindependent expressions and only resum the non-spinning part. In the remainder we find a logarithmic term which is factored out in a normalized form before the actual resummation (Eq. (38) in 41]). The normalization parameter $x_{\mathrm{LSO}}$ can be taken from [40], Eq. (3.23). Another factorization (Eqns. (39) and (44) in [41]) takes care of a putative pole in the expansion near the light ring, whose position $v_{\omega}$ light ring is given by Eq. (3.22) in [40] to $2 \mathrm{PN}$ accuracy. Thus, finally, one factor of the flux function is a plain Taylor expansion and is resummed with a neardiagonal (lower-diagonal) Padé approximation.

As a result we have a choice between two versions of $\mathrm{d} E / \mathrm{d} t$ that are to be inserted in Eq. (22). We will refer to the different fluxes as Taylor flux $(\overline{\mathrm{TF}})$ and Padé flux $(\mathrm{PF})$.

\section{Mapping EOB to ADMTT coordinates}

The link between ADM and EOB coordinates is given by a canonical transformation with generating function $g\left(\boldsymbol{q}, \boldsymbol{q}^{\prime}\right)$,

$$
p_{i} \mathrm{~d} q^{i}=p_{i}^{\prime} \mathrm{d} q^{i}+\mathrm{d} g\left(\boldsymbol{q}, \boldsymbol{q}^{\prime}\right),
$$

where $g_{\text {id }}\left(\boldsymbol{q}, \boldsymbol{q}^{\prime}\right)=0$ would correspond to the identity transformation [14, 16. Via a Legendre transformation we arrive at a more convenient generator $\tilde{G}\left(\boldsymbol{q}, \boldsymbol{p}^{\prime}\right)=$ $g\left(\boldsymbol{q}, \boldsymbol{q}^{\prime}\right)+p_{i}^{\prime} q^{\prime i}$ yielding

$$
p_{i} \mathrm{~d} q^{i}+q^{i} \mathrm{~d} p_{i}^{\prime}=\mathrm{d} \tilde{G}\left(\boldsymbol{q}, \boldsymbol{p}^{\prime}\right) .
$$

Decomposition into the identity map plus PN corrections,

$$
\begin{aligned}
\tilde{G}\left(\boldsymbol{q}, \boldsymbol{p}^{\prime}\right)= & q^{i} p_{i}^{\prime}+G\left(\boldsymbol{q}, \boldsymbol{p}^{\prime}\right), \\
G\left(\boldsymbol{q}, \boldsymbol{p}^{\prime}\right)= & \frac{1}{c^{2}} G_{1 \mathrm{PN}}\left(\boldsymbol{q}, \boldsymbol{p}^{\prime}\right)+\frac{1}{c^{4}} G_{2 \mathrm{PN}}\left(\boldsymbol{q}, \boldsymbol{p}^{\prime}\right) \\
& +\frac{1}{c^{6}} G_{3 \mathrm{PN}}\left(\boldsymbol{q}, \boldsymbol{p}^{\prime}\right),
\end{aligned}
$$

was the key to the determination of the generating function, which was accomplished by [14, 16]. Their result, which is not unique when including the $3 \mathrm{PN}$ level, reads

$$
\begin{aligned}
G_{1 \mathrm{PN}}\left(\boldsymbol{q}, \boldsymbol{p}^{\prime}\right)= & \left(\boldsymbol{q} \cdot \boldsymbol{p}^{\prime}\right)\left(a_{1} \boldsymbol{p}^{2}+\frac{a_{2}}{q}\right), \\
G_{2 \mathrm{PN}}\left(\boldsymbol{q}, \boldsymbol{p}^{\prime}\right)= & \left(\boldsymbol{q} \cdot \boldsymbol{p}^{\prime}\right)\left[b_{1} \boldsymbol{p}^{4}+\frac{1}{q}\left(b_{2} \boldsymbol{p}^{2}+b_{3}\left(\boldsymbol{n} \cdot \boldsymbol{p}^{\prime}\right)^{2}\right)\right. \\
& \left.+\frac{b_{4}}{q^{2}}\right], \\
G_{3 \mathrm{PN}}\left(\boldsymbol{q}, \boldsymbol{p}^{\prime}\right)= & \left(\boldsymbol{q} \cdot \boldsymbol{p}^{\prime}\right)\left[c_{1} \boldsymbol{p}^{\prime 6}+\frac{1}{q}\left(c_{2} \boldsymbol{p}^{4}+c_{3} \boldsymbol{p}^{2}\left(\boldsymbol{n} \cdot \boldsymbol{p}^{\prime}\right)^{2}\right.\right. \\
& \left.+c_{4}\left(\boldsymbol{n} \cdot \boldsymbol{p}^{\prime}\right)^{4}\right)+\frac{1}{q^{2}}\left(c_{5} \boldsymbol{p}^{\prime 2}+c_{6}\left(\boldsymbol{n} \cdot \boldsymbol{p}^{\prime}\right)^{2}\right) \\
& \left.+\frac{c_{7}}{q^{3}}\right],
\end{aligned}
$$

with

$$
\begin{aligned}
& a_{1}=-\frac{\nu}{2}, a_{2}=1+\frac{\nu}{2}, \\
& b_{1}=\frac{1}{8}\left(\nu+3 \nu^{2}\right), \quad b_{2}=\frac{1}{8}\left(2 \nu-5 \nu^{2}\right), \\
& b_{3}=\frac{1}{8}\left(8 \nu+3 \nu^{2}\right), \quad b_{4}=\frac{1}{4}\left(1-7 \nu+\nu^{2}\right), \\
& c_{1}=-\frac{1}{16}\left(1+3 \nu+5 \nu^{2}\right) \nu, \\
& c_{2}=-\frac{1}{16}\left(1+2 \nu-11 \nu^{2}\right) \nu, \\
& c_{3}=-\frac{1}{24}\left(12+48 \nu+23 \nu^{2}\right) \nu, \\
& c_{4}=\frac{1}{16}(24+7 \nu) \nu^{2}, \\
& c_{5}=-\frac{1}{16}\left(13-16 \nu+6 \nu^{2}\right) \nu, \\
& c_{6}=-\frac{1}{48}\left(115+116 \nu-26 \nu^{2}\right) \nu, \\
& c_{7}=-\left(\frac{1}{64} \pi^{2}+\frac{155}{24}\right) \nu+\frac{3}{8} \nu^{2}+\frac{1}{8} \nu^{3} .
\end{aligned}
$$

The sought-after transformation $\mathrm{EOB} \rightarrow \mathrm{ADM}$ is

$$
q^{i}=q^{i}-\frac{\partial G\left(\boldsymbol{q}, \boldsymbol{p}^{\prime}\right)}{\partial p_{i}^{\prime}}, \quad p_{i}=p_{i}^{\prime}+\frac{\partial G\left(\boldsymbol{q}, \boldsymbol{p}^{\prime}\right)}{\partial q^{i}},
$$


which gives an implicit relation that can be solved numerically by a root-finding algorithm. Another possibility to resolve the implicity is to repeatedly plug in the first equation in order to eliminate the unknown value $\boldsymbol{q}$ to sufficient PN order, yielding an explicit formula. By rearranging (36) we can calculate the inverse transformation, $\mathrm{ADM} \rightarrow \mathrm{EOB}$, in an analogous manner. The inverse transformation is convenient when discussing properties of the transformation and the associated errors, for example we can start with ADM data from the class of circularly orbiting $\mathrm{BH}$ binaries and study the result on the EOB side.

In order to illustrate the effect of the transformation, we consider the equal-mass case and choose, as a result from our initial data algorithm, the values

$$
\begin{aligned}
& \boldsymbol{q}=(10,0,0), \\
& \boldsymbol{p}=(-0.00394009,0.384516,0),
\end{aligned}
$$

for a separation of $D=10 M$ (with $M=c=G=1$ ). This translates to

$$
\begin{aligned}
& \boldsymbol{q}^{\prime}=(10.9445,0.00318625,0), \\
& \boldsymbol{p}^{\prime}=(-0.00397814,0.351514,0) .
\end{aligned}
$$

when using the explicit version of $\mathrm{ADM} \rightarrow \mathrm{EOB}$ at $3 \mathrm{PN}$ order. Note that the transformation mixes corresponding components of $(\boldsymbol{q}, \boldsymbol{p})$ and $\left(\boldsymbol{q}^{\prime}, \boldsymbol{p}^{\prime}\right)$, so the non-vanishing $y$-component of $\boldsymbol{p}$ causes a non-zero $y$-component of $\boldsymbol{q}^{\prime}$. The intermixture, however, is not a contradiction to the role of $\boldsymbol{q}$ and $\boldsymbol{q}^{\prime}$ as position variables and $\boldsymbol{p}$ and $\boldsymbol{p}^{\prime}$ as the conjugate momenta.

For equal masses, Fig. 1 illustrates the actual relation between both coordinate types in the range from $6 M$ to $14 M$, imposing non-eccentric momenta throughout. In particular, Fig. 1 a reveals that $|\boldsymbol{q}|$ and $\left|\boldsymbol{q}^{\prime}\right|$ (resp. $R$ and $R^{\prime}$ ) lie apart from each other by almost $1 M$.

However, the validity of the canonical transformation depends on the assumptions $\left|\boldsymbol{q}^{\prime}-\boldsymbol{q}\right| /|\boldsymbol{q}| \ll 1$ and $\left|\boldsymbol{p}^{\prime}-\boldsymbol{p}\right| /|\boldsymbol{p}| \ll 1$. The fulfillment of these conditions can be considered critical for the close binary separations under consideration, which might result in severe problems when using the spin and flux equations with EOB variables. Namely, as we have just found there is a significant difference between the radial distances $R$ and $R^{\prime}$, as well as some deviation in the direction vectors $N, \boldsymbol{N}^{\prime}$. These quantities appear in Eqns. (16)- 20). However, only (16) is incorporated in the initial data algorithm. Moreover, the transformation $\mathrm{d} \boldsymbol{p} \wedge \mathrm{d} \boldsymbol{q}=\mathrm{d} \boldsymbol{p}^{\prime} \wedge \mathrm{d} \boldsymbol{q}^{\prime}$ corresponds to $\mathrm{d} H \wedge \mathrm{d} t=\mathrm{d} H^{\prime} \wedge \mathrm{d} t^{\prime}$ and implies a change in the time scale [14. This also affects the orbital frequency $\omega$ which is used within the flux function (24). The quantity $\boldsymbol{L}$, however, is the same in both systems. The notion of spin vectors is supposed to be unaltered up to $1 \mathrm{PN}$ level, but modifications occur at higher PN orders. For a more detailed investigation we refer to the recently published reference 42, where the spin vectors are made subject of an additional canonical transformation.
Although we are aware of these issues, we note that applying suitable corrections in order to avoid these inconsistencies would be rather involved and would probably be unnecessarily sophisticated for our purpose. As already discussed, further issues arise anyway when translating the initial parameters obtained this way into the actual initial data (i.e. metric and curvature fields). In the end, the measured eccentricity decides whether our data - obtained under the specified simplifications - are appropriate.

\section{ALGORITHM FOR QUASI-SPHERICAL INITIAL DATA}

If we take into account only the conservative part of the orbital post-Newtonian dynamics, $H^{0}$, together with the leading-order spin-orbit interaction term, $H_{\mathrm{SO}}$, it is possible to find solutions which feature a constant coordinate separation $R$. These solutions are called spherical orbits. In general, this would no longer be true if we additionally included spin-spin coupling via $H_{\mathrm{SS}}$, although there might exist very special spin constellations for which a spherical orbit can be found. In the following we only consider spin-orbit coupling (with one exception mentionend below).

Imposing spin-orbit coupling we find two additional conserved quantities, the magnitude of the angular momentum $L=|\boldsymbol{L}|$ and

$$
\chi_{L}=\frac{c}{G M^{2}} \boldsymbol{S}_{\mathrm{eff}} \cdot \hat{\boldsymbol{L}}
$$

which gives the projection of the spins onto the direction of $\boldsymbol{L}$. Changing to polar coordinates with $\boldsymbol{L}$ pointing along the $z$-axis, the total Hamiltonian depends on only four quantities,

$$
H\left(R, P_{R}, L, \chi_{L}\right)=H^{0}\left(R, P_{R}, L\right)+2 \frac{G}{c^{2}} \frac{G M^{2}}{c} \frac{L \chi_{L}}{R^{3}},
$$

where we used the relation

$$
\boldsymbol{P}^{2}=P_{R}^{2}+P_{t}^{2}=P_{R}^{2}+\frac{P_{\varphi}^{2}}{R^{2}}
$$

and $P_{\varphi}=L$. Note that only the $z$-components of the spins enter. Beyond these considerations for the conservative part, radiation reaction will be included separately.

The challenge for the initial data algorithm is to find tangential and radial momentum of the objects for a given $R$ and $\chi_{L}$ such that no radial oscillations occur during evolution with the dissipative PN equations of motion.

Note that demanding a certain initial separation $R$ is a gauge dependent statement, which, however, is convenient for our purposes since the ADMTT gauge resembles the $1+\log$ /gamma-freezing gauge used in standard puncture evolutions. Under these circumstances, the algorithm takes a somewhat simpler form when using $R$ in order to specify the binary separation than 
a)

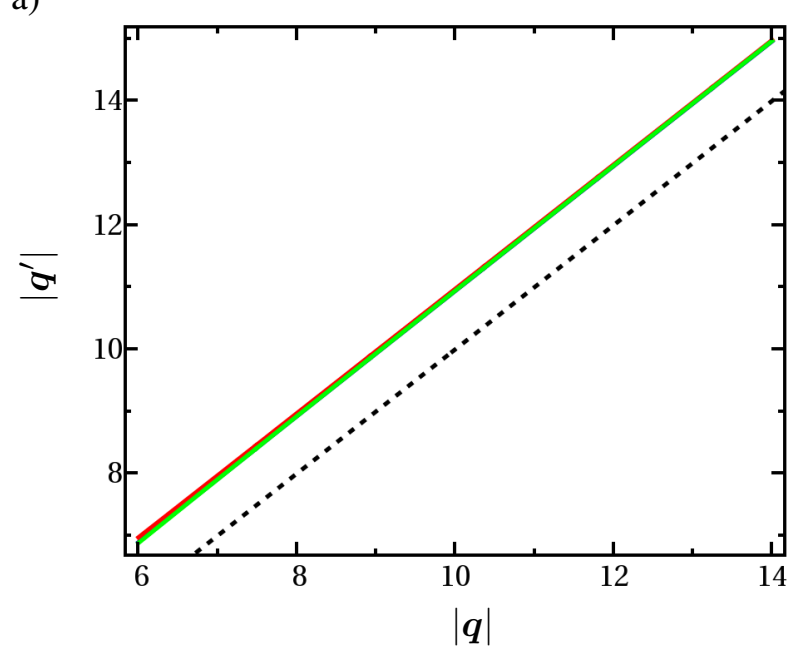

c)

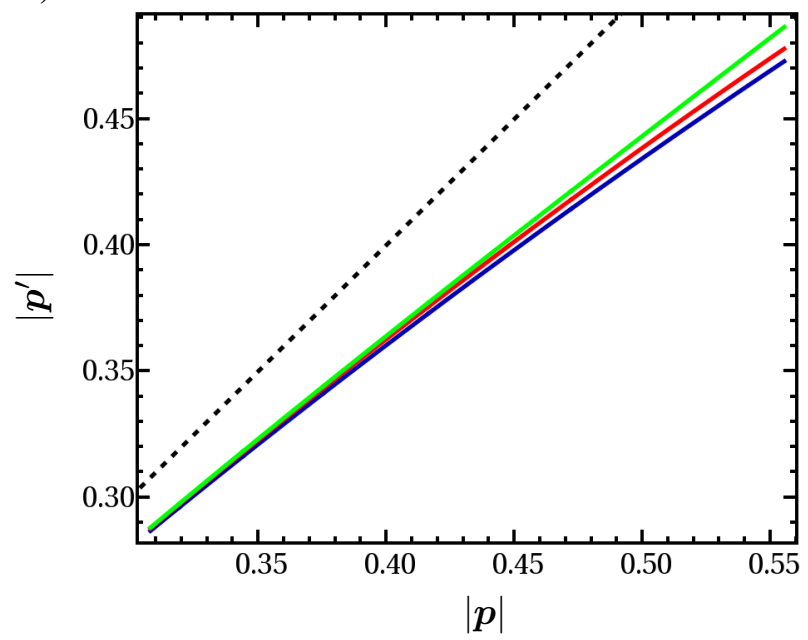

b)

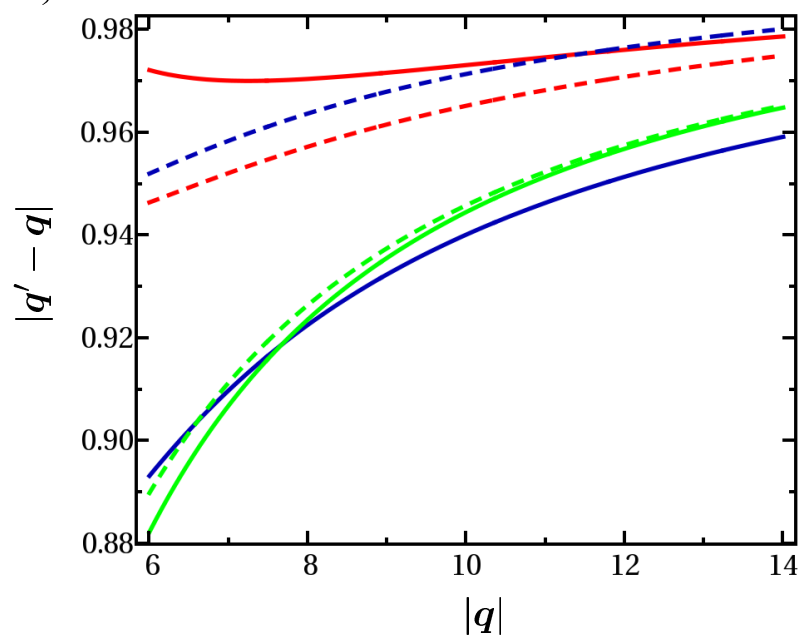

d)

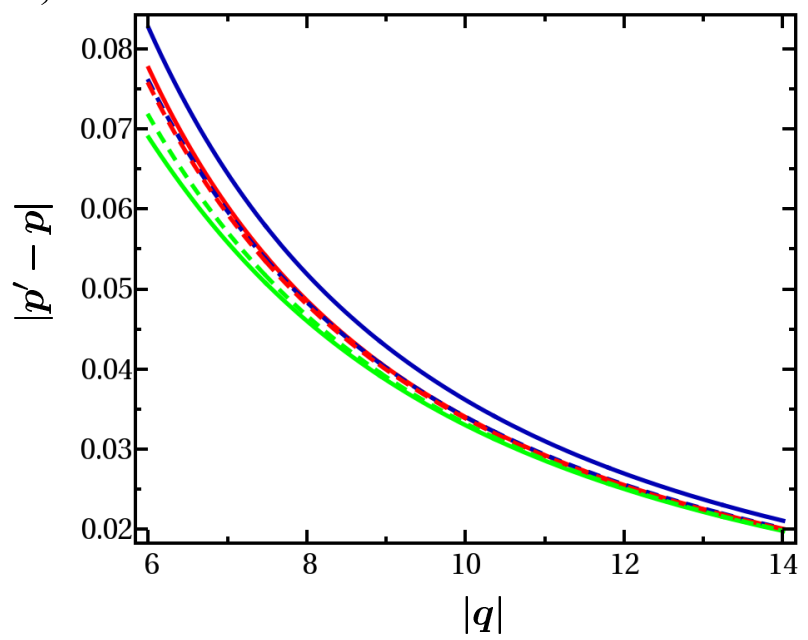

\begin{tabular}{|c|c|c|}
\hline $\begin{array}{l}\text { - } 1 \mathrm{PN} \text { (expl.) } \\
--1 \mathrm{PN} \text { (impl.) }\end{array}$ & $\begin{array}{l}-2 \mathrm{PN} \text { (expl.) } \\
--2 \mathrm{PN} \text { (impl.) }\end{array}$ &  \\
\hline
\end{tabular}

FIG. 1: (Color online) Character of the canonical transformation ADM $\rightarrow$ EOB for spherical initial data. The dotted line indicates the identity map $\boldsymbol{q}^{\prime}=\boldsymbol{q}$ and $\boldsymbol{p}^{\prime}=\boldsymbol{p}$. The plots on the right compare the explicit and implicit solution method of the transformation (36) and apparently there is convergence between the two versions with increasing PN order. However, convergence is not achieved conclusively, and the observed differences are used to define an error bar for the transformation. Since the explicit transformation is an approximation (truncated at finite order) to the implicit relation, it is less accurate.

when specifying the invariant orbital frequency $\omega$ instead, as originally done by Buonanno et al. 28. Furthermore, it eases the specification of spins, which can now be set with respect to the dynamical quantity $\hat{\boldsymbol{L}}_{0}=$ $\boldsymbol{X}_{0} \times \boldsymbol{P}_{0} /\left|\boldsymbol{X}_{0} \times \boldsymbol{P}_{0}\right|$ instead of the kinematical variable $\left[\hat{\boldsymbol{L}}_{\mathrm{N}}\right]_{0}=\boldsymbol{X}_{0} \times \dot{\boldsymbol{X}}_{0} /\left|\boldsymbol{X}_{0} \times \dot{\boldsymbol{X}}_{0}\right|$. We use the subscript "0" to stress that the initial value is meant.

The algorithm itself consists of two steps: (i) Determine $\left[P_{t}\right]_{0}$ as required for spherical orbits by considering the conservative motion only. (ii) Calculate the component $\left[P_{R}\right]_{0}$ by means of the energy flux in order to get a quasi-spherical inspiral.
In the first step of the procedure we determine $\left[P_{t}\right]_{0}$, or equivalently $L_{0}=R_{0} \cdot\left[P_{t}\right]_{0}$, by demanding constant radius,

$$
0 \stackrel{!}{=}[\dot{R}]_{0}=\left[\frac{\partial H\left(R, P_{R}, L, \chi_{L}\right)}{\partial P_{R}}\right]_{0} .
$$

Since $P_{R}$ enters quadratically into $H$ this is equivalent to

$$
\left[P_{R}\right]_{0}=0 .
$$

The value of $\chi_{L}$ is known and thus there is only one remaining degree of freedom, namely the value of $L_{0}$. It 
is fixed by demanding

$$
0 \stackrel{!}{=}\left[\dot{P}_{R}\right]_{0}=-\left[\frac{\partial H\left(R, P_{R}, L, \chi_{L}\right)}{\partial R}\right]_{0},
$$

a condition which has to be solved numerically. A starting value for an iteration procedure can be provided by the $3 \mathrm{PN}$-accurate tangential momentum for circular orbits of non-spinning objects,

$$
\begin{aligned}
P_{t}^{3 \mathrm{PN}}= & \mu\left[\left(\frac{G M}{D}\right)^{1 / 2}+\frac{1}{c^{2}} 2\left(\frac{G M}{D}\right)^{3 / 2}+\frac{1}{c^{4}} \frac{1}{16}(42\right. \\
& -43 \nu)\left(\frac{G M}{D}\right)^{5 / 2}+\frac{1}{c^{6}} \frac{1}{128}\left[480+\left(163 \pi^{2}\right.\right. \\
& \left.\left.-4556) \nu+104 \nu^{2}\right]\left(\frac{G M}{D}\right)^{7 / 2}\right]
\end{aligned}
$$

a formula given in [10.

The second step incorporates radiation reaction and consequently a non-vanishing $\dot{R}$. Assuming adiabatic inspiral, that is a sequence of spherical orbits whose shrinkage in radius is determined by the energy flux, one finds

$$
[\dot{R}]_{0}=\frac{[\mathrm{d} E / \mathrm{d} t]_{0}}{\left[(\mathrm{~d} E / \mathrm{d} R)_{\mathrm{sph}}\right]_{0}},
$$

where $\mathrm{d} E / \mathrm{d} t$ is given by Eq. 24 or its Padé resummation. Here the knowledge of the initial orbital frequency $\omega_{0}$ is required and can be provided by the evolution equation

$$
\omega_{0}=[\dot{\varphi}]_{0}=\left[\frac{\partial H}{\partial P_{\varphi}}\right]_{0}=\left[\frac{\partial H}{\partial L}\right]_{0} .
$$

The initial conditions used in the RHS of this equation, i.e. the components of the momentum, are taken from the first step, in particular $\left[P_{R}\right]_{0}=0$. The other quantity appearing in (46) is the energy difference between neighboring spherical orbits and can be calculated as

$$
\begin{aligned}
\left(\frac{\mathrm{d} E}{\mathrm{~d} R}\right)_{\mathrm{sph}}= & \frac{\partial H}{\partial R}\left(\frac{\mathrm{d} R}{\mathrm{~d} R}\right)_{\mathrm{sph}}+\frac{\partial H}{\partial P_{R}}\left(\frac{\mathrm{d} P_{R}}{\mathrm{~d} R}\right)_{\mathrm{sph}} \\
& +\frac{\partial H}{\partial L}\left(\frac{\mathrm{d} L}{\mathrm{~d} R}\right)_{\mathrm{sph}}+\frac{\partial H}{\partial \chi_{L}}\left(\frac{\mathrm{d} \chi_{L}}{\mathrm{~d} R}\right)_{\mathrm{sph}} .
\end{aligned}
$$

The first two terms vanish, because for spherical orbits $\partial H / \partial R=-P_{R}=0$ holds and consequently $\mathrm{d} P_{R}=0$, too. The other two summands are problematic in that there appear the unknowns $(\mathrm{d} L / \mathrm{d} R)_{\mathrm{sph}}$ and $\left(\mathrm{d} \chi_{L} / \mathrm{d} R\right)_{\mathrm{sph}}$. We are able to substitute the first of them by considering

$$
\begin{aligned}
0 & =\left(\frac{\mathrm{d}}{\mathrm{d} R} \frac{\partial H}{\partial R}\right)_{\mathrm{sph}} \\
& =\frac{\partial^{2} H}{\partial R^{2}}+\frac{\partial^{2} H}{\partial L \partial R}\left(\frac{\mathrm{d} L}{\mathrm{~d} R}\right)_{\mathrm{sph}}+\frac{\partial^{2} H}{\partial \chi_{L} \partial R}\left(\frac{\mathrm{d} \chi_{L}}{\mathrm{~d} R}\right)_{\mathrm{sph}},
\end{aligned}
$$

where we have omitted vanishing terms. Rearranging for $(\mathrm{d} L / \mathrm{d} R)_{\mathrm{sph}}$ and inserting into 48$)$ we obtain

$$
\begin{aligned}
&\left(\frac{\mathrm{d} E}{\mathrm{~d} R}\right)_{\mathrm{sph}}=-\frac{\left(\frac{\partial H}{\partial L}\right)\left(\frac{\partial^{2} H}{\partial R^{2}}\right)}{\left(\frac{\partial^{2} H}{\partial R \partial L}\right)}+\left[\left(\frac{\partial H}{\partial \chi_{L}}\right)\right. \\
&\left.-\frac{\left(\frac{\partial H}{\partial L}\right)\left(\frac{\partial^{2} H}{\partial R \partial \chi_{L}}\right)}{\left(\frac{\partial^{2} H}{\partial R \partial L}\right)}\right]\left(\frac{\mathrm{d} \chi_{L}}{\mathrm{~d} R}\right)_{\mathrm{sph}}, \\
& {\left[\left(\frac{\mathrm{d} E}{\mathrm{~d} R}\right)_{\mathrm{sph}}\right]_{0} \approx-\frac{\left[\left(\frac{\partial H}{\partial L}\right)\right]_{0}\left[\left(\frac{\partial^{2} H}{\partial R^{2}}\right)\right]_{0}}{\left[\left(\frac{\partial^{2} H}{\partial R \partial L}\right)\right]_{0}} . }
\end{aligned}
$$

The second part has been neglected as $\chi_{L}$ is supposed to be an almost conserved quantity even though radiation reaction is turned on. According to the analysis of 28 the corresponding error is of $3 \mathrm{PN}$ order. With $[\dot{R}]_{0}$ in hand we can exploit the abovementioned quadratic appearance of $P_{R}$ within the Hamiltonian to obtain

$$
\left[P_{R}\right]_{0}=\frac{[\dot{R}]_{0}}{\left[\frac{1}{P_{R}} \frac{\partial H}{\partial P_{R}}\right]_{0, P_{R} \rightarrow 0}}=\frac{[\dot{R}]_{0}}{2\left[\frac{\partial H}{\partial\left(P_{R}^{2}\right)}\right]_{0}} .
$$

Hence, the algorithm gives $\left[P_{t}\right]_{0}$ and $\left[P_{R}\right]_{0}$ in a coordinate system where $\hat{\boldsymbol{L}}_{0}$ (by definition) represents the $z$-axis. We omit the subscript " 0 " from here on as it is clear that we refer to initial data. Furthermore we use the labels $R$ and $D$, denoting the binary separation, interchangeably.

The procedure just described is valid for both types of Hamiltonians. For the Taylor-expanded version we immediately obtain the final result in ADMTT gauge. Employing the EOB approach on the other hand is rather more involved. We shall briefly illustrate why.

First, recall that in the EOB case the above algorithm is applied for the primed variables, which represents a simplification as we discussed above. However, in the end we want to obtain the result in ADMTT coordinates. Moreover, we wish to specify the ADMTT distance, i.e. $\boldsymbol{X}$, at which the initial momentum is to be determined. The canonical transformation ADM $\leftrightarrow$ EOB only mediates between the whole set of variables $(\boldsymbol{X}, \boldsymbol{P}) \leftrightarrow\left(\boldsymbol{X}^{\prime}, \boldsymbol{P}^{\prime}\right)$, thus providing the corresponding value of $\boldsymbol{X}^{\prime}$ for use within the algorithm would already require the knowledge of the sought-after solution $\boldsymbol{P}$. Hence, an additional step becomes necessary, for example a shooting method, to achieve a match with the given ADM distance. We take care of this for our actual initial data computations in order to compare numerical results at identical values of $\boldsymbol{X}$.

Translating EOB results to ADM coordinates involves a certain degree of inaccuracy because the transformation is only known approximately (to $3 \mathrm{PN}$ order). Table円gives some conservative, rough error estimates based on PN convergence as shown in Fig. 1. The estimates already include the error contribution that would arise 


\begin{tabular}{cccccccccc}
\hline \hline Mass ratio & \multicolumn{2}{c}{$R_{0} / M$} & & & & & & & \\
& 6 & 7 & 8 & 9 & 10 & 11 & 12 & 13 & 14 \\
\hline $1: 1$ & 3.1 & 1.8 & 1.1 & 0.78 & 0.55 & 0.40 & 0.30 & 0.23 & 0.18 \\
$4: 1$ & 2.2 & 1.2 & 0.74 & 0.49 & 0.34 & 0.25 & 0.19 & 0.14 & 0.11 \\
$10: 1$ & 0.99 & 0.55 & 0.34 & 0.21 & 0.15 & 0.11 & 0.08 & 0.06 & 0.05 \\
\hline \hline
\end{tabular}

TABLE I: Estimate of the relative error in percent for EOB-based initial data $P_{t}$ and $P_{R}$ at various binary separations and mass ratios.

from matching with a given ADM separation when applying the shooting procedure. Apparently, the transformation can be regarded more accurate for larger separations and higher mass ratios. We conclude this section by presenting some examples for the orbital parameters $P_{t}$ and $P_{R}$ obtained by the algorithm. Figs. 2 and 3 show initial parameters for an equal-mass binary without spins. Significant deviations between the various combinations, that is combining one of the Hamiltonians, either Taylor-expanded $(\mathrm{TH})$ or EOB Hamiltonian $(\mathrm{EH})$, with one version of the flux function, either Taylor-expanded (TF) or Pade-resummed (PF), can be observed only at small separations. Some dependence on the choice of the flux function is observed in Fig. 3, although the result depends more strongly on the choice of Hamiltonian. This effect becomes more pronounced for higher mass ratios, see Fig. 4. The plots clearly indicate the expected degradation of the $\mathrm{TH}$ parameters for small separations, which is caused by a zero transition of the second derivative $\partial^{2} H / \partial R^{2}$ in Eq. (50), while the first derivative and thus $\partial^{2} H /(\partial R \partial L)$ does not vanish. The resulting pole moves outwards to larger separations and spoils the data as the mass ratio increases. We would conclude that the accompanying drift in the $P_{t}$ curves is also a result of the Taylor Hamiltonian's behavior. Clearly, it would not make sense to use TH data in the vicinity of the pole, Fig. 4 d.

The EOB data on the other hand appear sound and remain close to the $P_{t}$ values of [10] (our Eq. (45)), but might be affected by the transformation error. In fact, in the equal-mass case there is concern that the transformation error exceeds the difference to the $\mathrm{TH}$ data. However, since the error goes down at higher mass ratios we expect the EOB data to be superior in these cases.

We examine the data's behavior in the presence of spins at the end of Section IV

\section{NUMERICAL RESULTS}

For a specific set of parameters, comprising masses, initial separation and spins, the initial data algorithm can give us tangential and radial momentum of a relative particle. The actual initial configuration, consisting of the individual locations and momenta of the black holes, can be obtained by placing the bodies appropriately in a coordinate frame by setting $\boldsymbol{P}_{1}=-\boldsymbol{P}_{2}=\boldsymbol{P}$ and e.g. letting the center of mass coincide with the origin. We are also free to apply rotations.

As discussed the input parameters $m_{a}, \boldsymbol{X}_{a}, \boldsymbol{P}_{a}, \boldsymbol{S}_{a}$ are formulated in (almost) ADMTT gauge whereas the BAM code uses a different spatial metric $\gamma_{i j}=\psi_{0}^{4} \tilde{\gamma}_{i j}$ with a conformally flat background metric $\tilde{\gamma}_{i j}=\delta_{i j}$ on a maximal slice $(K=0)$ (so-called black hole puncture data [13). In previous work it was found numerically that the two gauges are rather close to each other (e.g. 10. 21). However, it is by no means trivial nor entirely understood why PN-non-eccentric initial parameters should produce non-eccentric orbits in full GR. First, the process of translating the initial parameters to the actual initial data involves the solution of the Hamiltonian constraint, which, for example, alters the mass parameters, cf. Table V Second, the modelled configuration lacks the gravitational-wave content which would be there if the binary had reached this state in a physically realistic way. The constraint solution process and the ad-hoc specification of data at a given time result in artificial waves in the initial data, which e.g. affects the black-hole positions in the beginning of the simulation. Third, the gauge (lapse and shift) evolves in the starting phase, settling down to the final values after about $50 \mathrm{M}$ of coordinate time. These effects may not only outweigh the hard-won subtle differences between THTF, THPF, EHTF and EHPF initial data, but also make it difficult to reliably assess the quality of these data.

\begin{tabular}{ccr}
\hline \hline Mass ratio & $h_{\min , i} / M_{i}$ & $r_{\text {out }} / M$ \\
\hline $1: 1$ & $3 / 64$ & 774 \\
$1: 2$ & $3 / 56$ & 2067 \\
$1: 4$ & $1 / 96$ & 1236 \\
\hline \hline
\end{tabular}

TABLE II: Grid setups of the different types of numerical simulations. The index $i$ enumerates the black holes $(i=$ 1,2) and $M=M_{1}+M_{2}$ is the total mass of the system. $h_{\min , i}$ is the resolution of the finest grid covering the $i^{\text {th }}$ black hole. $r_{\text {out }}$ is the position of the outer boundary. All results throughout the paper corresponding to the same mass ratio (but e.g. different initial separations or spins) were obtained with the setup given here.

For the purpose of the latter we investigated several approaches for measuring the eccentricity of the inspiralling binary's orbit. Following the ideas of [9, 21, 43, 44, we employ two approaches based on the evolution of the separation $D(t)$ and the orbital frequency $\omega(t)$. The first 

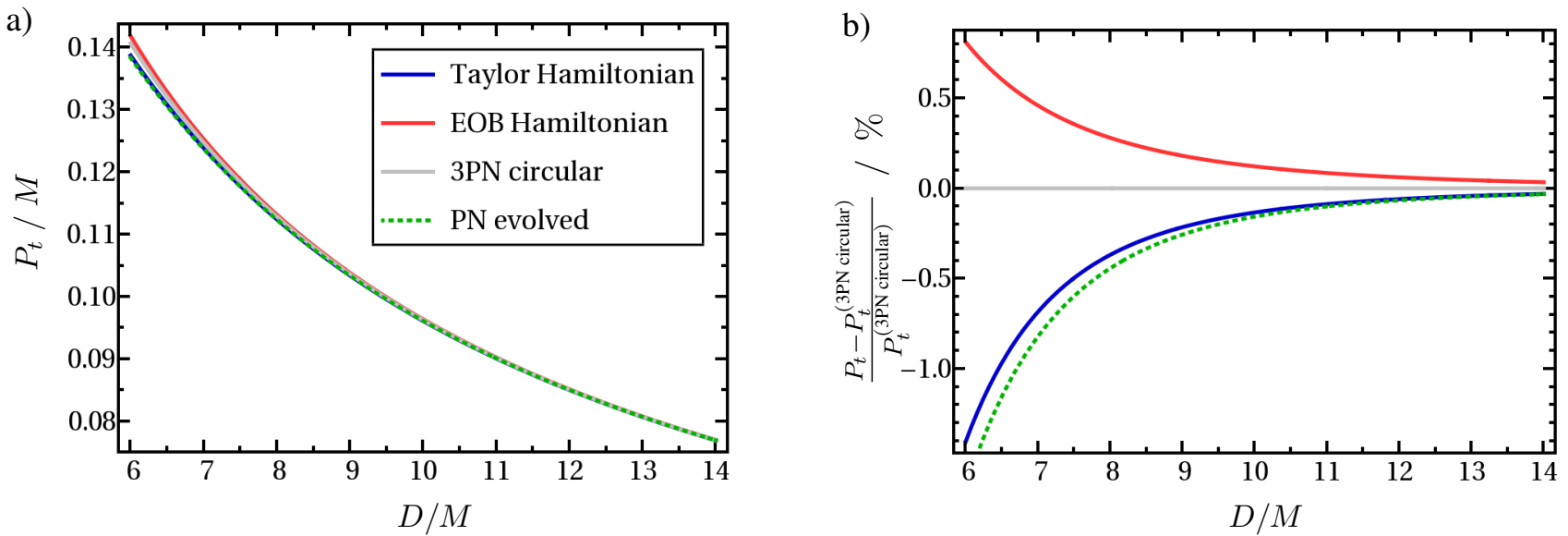

FIG. 2: (Color online) Tangential momentum obtained with the initial data algorithm for an equal-mass binary $(M=1$, no spins). Also shown are reference functions "3PN circular" from [10] (cf. Eq. 445) and "PN evolved" from 21. The right panel gives the deviation in percent with respect to the $3 \mathrm{PN}$-circular curve.
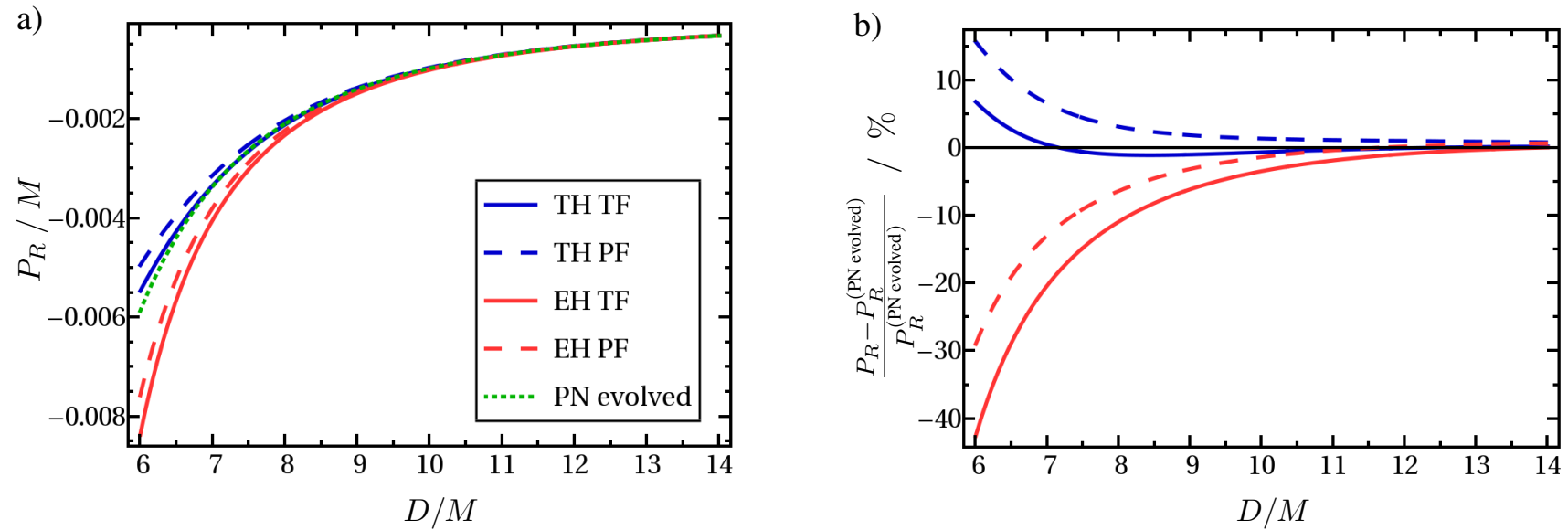

FIG. 3: (Color online) Radial momentum obtained with the initial data algorithm for an equal-mass binary ( $M=1$, no spins). Compared are combinations of the two Hamiltonians (Taylor or EOB) with the two possible flux functions (Taylor or Padé). As expected, the THTF curve almost coincides with the reference function "PN evolved" from [21].

\begin{tabular}{ccccccc}
\hline \hline Mass ratio & $D / M$ & Data type & $P_{t} / M$ & $P_{R} / 10^{-3} M$ & Interval $/ M$ & Eccentricity \\
\hline \multirow{2}{*}{$1: 1$} & \multirow{2}{*}{11} & THTF & 0.090110 & -0.71442 & $300 \ldots 600$ & 0.002 \\
& & EHPF & 0.090266 & -0.71489 & $300 \ldots 600$ & 0.003 \\
\hline $1: 1$ & 7 & THTF & 0.123863 & -3.34124 & $60 \ldots 120$ & 0.005 \\
& & THPF & $\prime \prime$ & -3.13589 & $60 \ldots 120$ & 0.005 \\
& & EHTF & 0.125288 & -4.04062 & $80 \ldots 150$ & 0.005 \\
& & EHPF & " & -3.79255 & $80 \ldots 150$ & 0.005 \\
\hline $2: 1$ & 10 & THTF & 0.085615 & -0.79486 & $250 \ldots 400$ & 0.002 \\
& & EHTF & 0.085753 & -0.80701 & $250 \ldots 400$ & 0.003 \\
\hline $4: 1$ & 10 & THTF & 0.061921 & -0.43393 & $300 \ldots 700$ & 0.004 \\
& & EHPF & 0.061883 & -0.42106 & $300 \ldots 700$ & 0.0025 \\
\hline \hline
\end{tabular}

TABLE III: Simulations of black hole binaries without spin for equal and unequal masses. A local eccentricity measurement was performed in the time interval given. The uncertainty in the eccentricity is estimated to be \pm 0.001 .

consists of comparing the actual with an ideal (quasicircular) inspiral curve $D_{c}(t)$ or $\omega_{c}(t)$, which is obtained by averaging over the eccentricity oscillations us- ing an appropriate fit function (also see Fig. 9). The resulting eccentricities are referred to as $e_{D}$ and $e_{\omega}$. Specifically, we employ a model function according to 

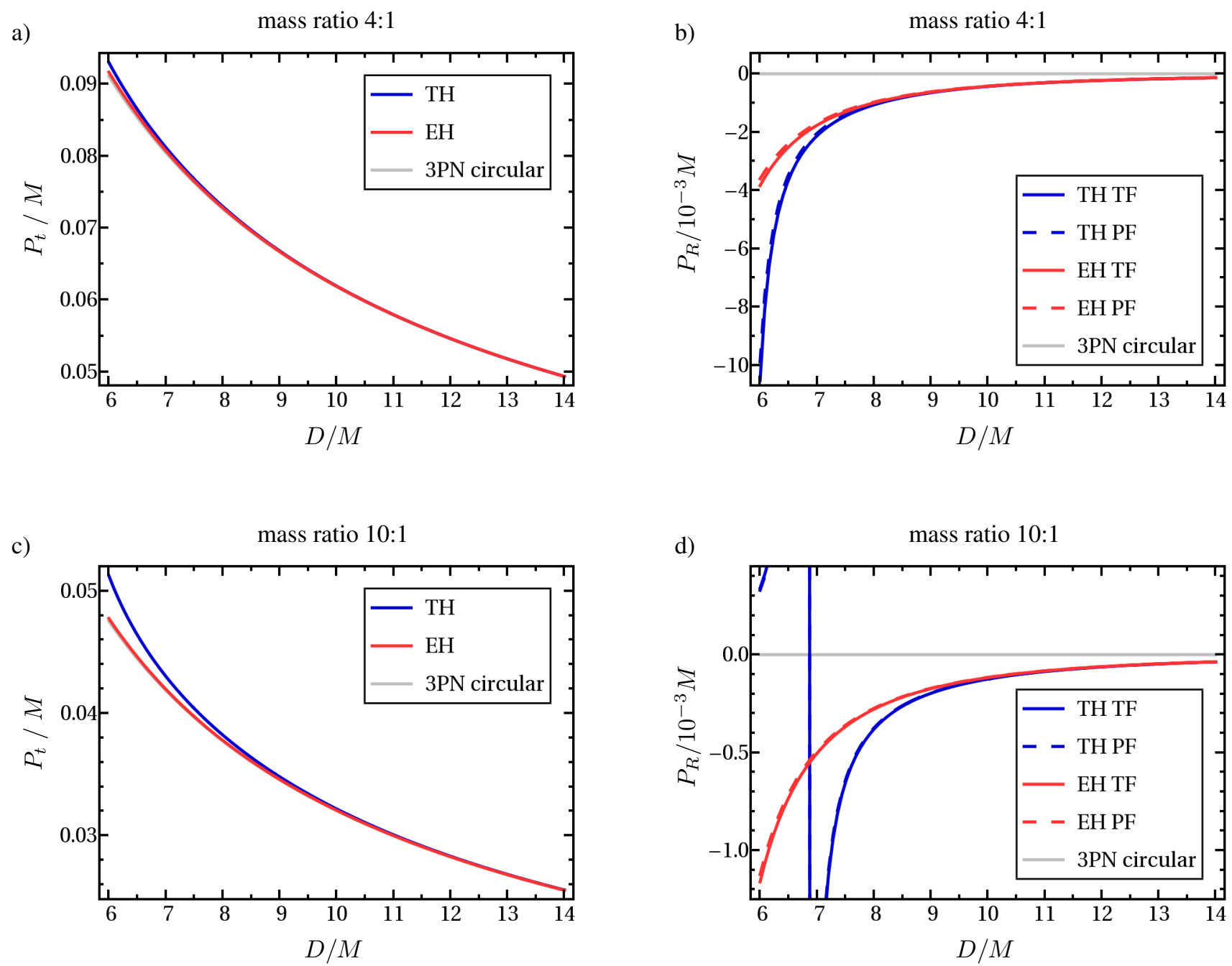

FIG. 4: (Color online) Behavior of Taylor-expanded and EOB initial data for higher mass ratios $\left(M=1\right.$, no spins). For $P_{t}$, $\mathrm{EH}$ and "3PN circular" almost coincide, while $P_{R}=0$ for "3PN circular".

$D_{c}(t)=\sum_{i=1}^{4} a_{i}\left(t_{M}-t\right)^{i / 2}$ and eccentricity is computed as $e_{D}(t)=\left[D(t)-D_{c}(t)\right] / D_{c}(t)$ 21. The time $t_{M}$ entering the formula for the ideal separation $D_{c}(t)$ is the merger time which in our case is estimated by the fitting procedure. The resulting value agrees very well with results from different methods for measuring the merger time, for example the time when the amplitude of $\Psi_{4}$ reaches its maximum. As a model function for the frequency we use a fourth order polynomial, $\omega_{c}(t)=\sum_{i=0}^{4} b_{i} t^{i}$, following [45]. In this case, the eccentricity is calculated via $e_{\omega}(t)=\left[\omega(t)-\omega_{c}(t)\right] / 2 \omega_{c}(t)$. The fit to the model function only works well if we exclude initial gauge adjustments and it fails at merger time $\left(D_{c}\left(t=t_{M}\right)=0\right.$ in the $D$-method and the fourth order polynomial ceases to capture the behavior of the frequency in the $\omega$-method). We therefore have to choose an appropriate time interval for the averaging procedure. Figures 58 show graphs for eccentricities varying with time resulting from the $D$-method according to the for- mula given above. The global extremum of each curve, usually located at early times, is then used to determine a time-independent eccentricity value of the data which will serve to compare different runs with each other.

A second method tries to capture the oscillations themselves by presuming some functional dependence, i.e. a sinusoidal fluctuation on a certain background function like $\dot{D}(t)=A_{1}+A_{2} t+B \sin \left(\Omega_{0} t+\phi_{0}\right)$, where $A_{1}, A_{2}, B, \Omega_{0}$ and $\phi_{0}$ are fit parameters. This works best for the time derivatives $\dot{D}(t)$ and $\dot{\omega}(t)$ instead of the original functions. The resulting eccentricity can be estimated to be $e_{\dot{D}}(t) \approx-B \cos \left(\Omega_{0} t+\phi_{0}\right) / \Omega_{0} D(t)$. The procedure is equally applicable to the derivative of the frequency curve, $\dot{\omega}(t)$, and leads to an eccentricity denoted by $e_{\dot{\omega}}(t)$. Since the assumed "capture" functions are only able to trace the curves $\dot{D}(t)$ and $\dot{\omega}(t)$ locally (in a very short time interval), eccentricities computed with this approach turned out to be less useful for our purpose. 
All in all, the different methods $\left(e_{D}, e_{\omega}, e_{\dot{D}}\right.$ and $\left.e_{\dot{\omega}}\right)$ do not always give consistent results for very low eccentricities, for which the quality of the fit function becomes decisive. Eccentricity values given in the present paper are based on $e_{D}$, with error estimates based on the variations in the extrema of $e_{D}$.

Numerical evolutions were performed with the BAM code [10, 46] which uses the BSSN formulation of the Einstein equations 47, 48 and the method of moving punctures [49, 50]. Spatial derivatives are $6^{\text {th }}$ order accurate [51] and a $4^{\text {th }}$ order Runge-Kutta scheme is employed for time stepping. We use " $1+\log$ " slicing for determining the lapse function [52] and the "000"-version of the $\tilde{\Gamma}$-driver shift condition [53, 54]. In order to resolve the black hole regions as well as the wave zone sufficiently well, mesh refinement with nested boxes is used. Details of the grid setups used for numerical simulations are given in Table II. Note the choice of resolutions for the black holes: the number of refinement boxes is chosen for each black hole individually in order to obtain the same effective resolution $h_{\min }$ for both of them.

\section{A. Numerical results for different mass ratios and vanishing spin}

Table III summarizes the outcome of eccentricity measurement for various simulations with equal and unequal masses without spin. The initial separation was partially chosen to allow direct comparison with 21, 25. Fig. 5 shows the time dependence of $e_{D}$ for mass ratio 4:1, giving a visual impression of the oscillations of the inspiralling orbit, while Fig. 6 shows results for 1:1.

We draw the following conclusions from the numerical results. Our techniques for measuring eccentricity give compatible results in most cases, but in some cases the accuracy of \pm 0.001 is not quite sufficient to reliably resolve the smallish differences. Unless the eccentricities deviate by amounts larger than the uncertainties we cannot name one of the data the better choice. In particular, we examined the consequences of the choice of flux for the smallest initial separation $D=7 M$ case, where the differences should be largest. At the given level of accuracy we cannot discern a significant difference between the TF and PF methods.

For initial separations of $D=10 M$ and $D=11 M$, the eccentricity ranges from $e=0.002$ to 0.004 for comparatively small variations of about $1 \%$ in $P_{t}$ and $2 \%$ in $P_{R}$. This reflects the high sensitivity of the eccentricity to small changes in the momenta. The minimal eccentricity obtainable by the method is about $e=0.0025$ for mass ratios from 1:1 to 4:1.

For smaller initial binary separation the PN initial data become less appropriate since the underlying equations approach their limit of validity. Moreover, the deviation in the gauges, that is ADMTT gauge on the PN side versus maximal slicing in the numerical simulation, grows. The numerical example for $D=7 M$ shows that the ec- a)

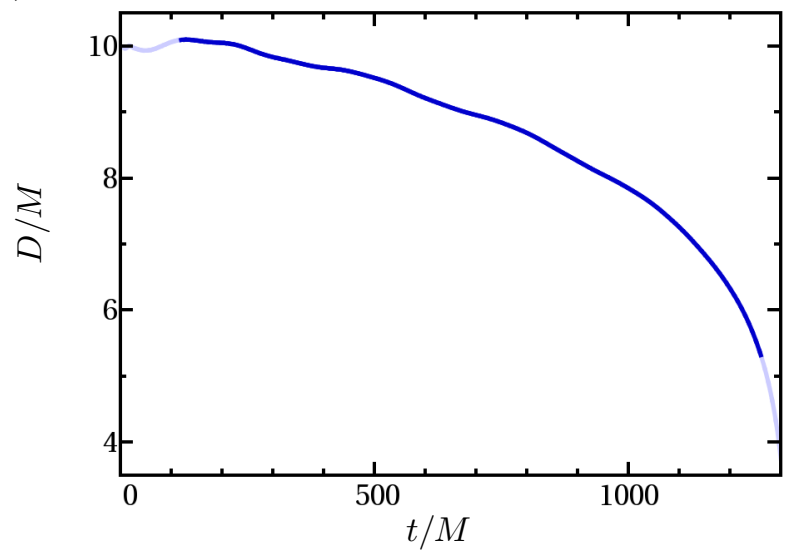

b)

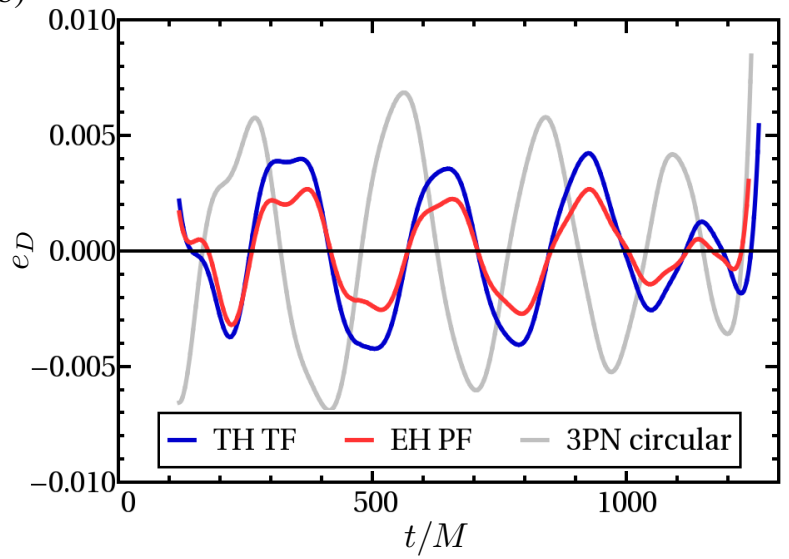

FIG. 5: (Color online) Dynamics and eccentricity for mass ratio $4: 1(D=10 M)$. Panel (a) shows the oscillating decrease of the binary separation for the THTF data. The darker part of the line indicates the time interval used for the fit required for $e_{D}$. In panel (b) the eccentricity is compared between Taylor-expanded and EOB-Hamiltonian data.

centricity grows to $e=0.005$ for equal masses, which is twice as large as for $D=11 M$. Considering how close $D=7 M$ is to the merger, it is surprising how well the PN initial parameters work in this context.

Going to higher mass ratios the putative advantage of EH data first seems to show at 4:1, although the difference in initial momentum to TH data is quite small. Given the available data, the smaller eccentricity might be accidental, but we believe there is a systematic trend based on corresponding results for spinning binaries. (Numerical simulations at 10:1 have recently become available [55], so the behavior for increasing mass ratios could now be investigated further.)

Utilizing the same Hamiltonian, our TH data are expected to give results similar to the $\mathrm{PN}$-evolved data, and this is indeed the case. For example, 21] obtained $e_{D}=0.002 \pm 0.001$ for the $1: 1$ case starting at $D=11 M$. Our TH results are also consistent with the outcome of $\mathrm{PN}$-evolved data at higher mass ratios, for example 25] found $e=0.003$ for mass ratio 2:1 and $e=0.005$ for the $4: 1$ case at $D=10 M$. 


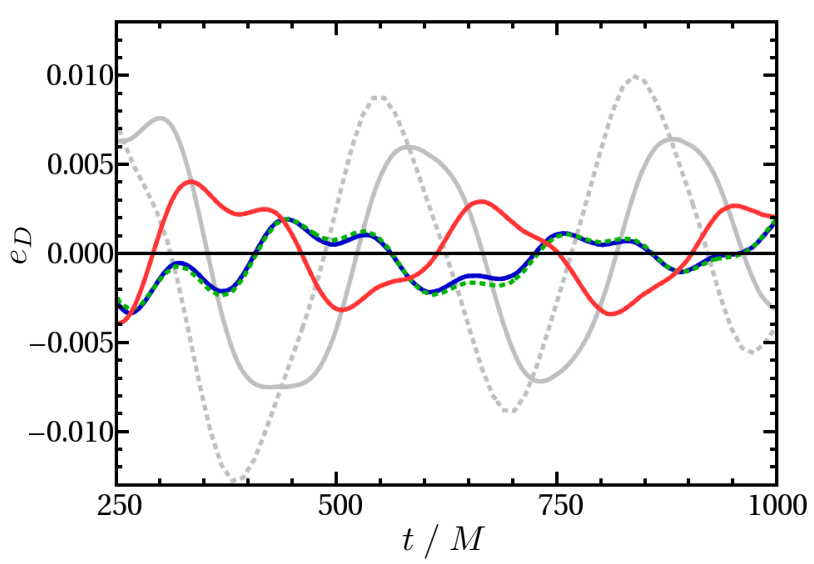

\begin{tabular}{|c|c|c|}
\hline - TH TF & ... PN evolved & - 3PN circular \\
\hline - EHPF & & $=-2 \mathrm{PN}$ circular \\
\hline
\end{tabular}

FIG. 6: (Color online) Eccentricity for mass ratio $1: 1(D=$ $11 M)$. Compared are runs for PN-circular data, PN-evolved data, and the new THTF and EHPF data. The THTF run is very similar to the $\mathrm{PN}$-evolved run as expected, while for $2 \mathrm{PN}$ circular data the eccentricity is up to a factor of five larger.

In 21, which introduced the PN evolution method to obtain initial parameters with reduced eccentricity, the PN evolutions were started with 3PN circular parameters of [10], and the result was compared to numerical simulations starting from $2 \mathrm{PN}$ (one order lower) circular parameters based on [22, 23]. The PN-evolved data showed an improvement by a factor of up to five in the eccentricity compared to the $2 \mathrm{PN}$ circular parameters for mass ratio 1:1. In Fig. 6 we compare the result for 1:1 for $2 \mathrm{PN}$ and $3 \mathrm{PN}$ circular parameters, the PN-evolved parameters, and the new THTF and EHPF parameters. The THTF run is very similar to the PN-evolved run as already pointed out. As stated in Table [II], the EHPF parameters result in about $50 \%$ larger eccentricity (for equal masses). For numerical simulations starting from the $3 \mathrm{PN}$ instead of $2 \mathrm{PN}$ circular parameters the improvement is less, about a factor of two for mass ratio 4:1 and 1:1 as shown in Figs. 5 and 6. Incidentally, note that the 2PN circular parameters are based on harmonic coordinates while the $3 \mathrm{PN}$ circular parameters refer to ADMTT.

Our final comment on the vanishing spin case is that, as shown in Figs. 5 and 6 , there still is a distinctive orbital eccentricity for mass ratio 4:1, while for $1: 1$ the eccentricity is reduced to a level where the oscillations are less clearly associated with the orbital motion. At least for $4: 1$ there seems to be room for improvement.

\section{B. Numerical results for equal masses and non-vanishing spin}

Let us now turn to spinning black hole binaries for equal masses. We would like to emphasize that as soon as spins are present eccentricity is inevitable. This is due to spin-spin interaction and can be seen from the PN equations of motion (3), which predict a precession of the spins with a frequency determined by (18). This precession influences the orbital motion via Eq. (16). Moreover, we find an even more direct impact on the orbital motion as a consequence of the $R$-dependence in (18) which directly affects the evolution of $\boldsymbol{P}$ in $(2)$. The latter mechanism is also relevant when both spins are parallel to the orbital angular momentum. Despite the constancy of $\boldsymbol{L}=\boldsymbol{X} \times \boldsymbol{P}$ in these cases (ignoring radiation for the time being), the orbital variables $\boldsymbol{X}$ and $\boldsymbol{P}$ would still be allowed to exhibit the corresponding oscillations.

Table IV]a illustrates this issue for an evolution with the conservative PN equations. (This is different from the PN-evolved parameters we discuss elsewhere which include flux terms.) The test case consists of an equal-mass binary with spin settings according to $\boldsymbol{S}_{i}=$ $G m_{i} / c^{2} \cdot \chi \hat{\boldsymbol{L}}$. We applied suitable initial data from the above algorithm which would give circular orbits if only $H_{\mathrm{SO}}$ was involved (in particular we dropped the $\left[P_{R}\right]_{0}$ component). The observed radial fluctuations are of orbital frequency (when taking periastron advance into account) and can be explained by means of Eqns. (18)(20). Since neither $\boldsymbol{L}$ nor the spins precess in the current constellation, the spin-spin contribution just represents a spherically symmetric deformation term $H_{\mathrm{SS}}=$ const $\cdot R^{-3}$. Due to its simplicity in this particular case the correction could in principle be taken into account when computing the initial parameters. For general spin configurations, however, this is not advisable, so we do not pursue this idea here but return to it later. Instead we can regard the numbers in Table [IV] a as an estimate of the minimal eccentricities to be achieved in fully numerical simulations. (For $\chi=0$, the eccentricity is analytically zero, and the small deviations from zero in Table IV a are numerical error of the integration method.)

The aligned and anti-aligned scenarios were explored with several runs. The main purpose was to find out which of the data type (THTF, THPF, EHTF, EHPF) leads to minimal eccentricity. The observation that the choice of the flux does not play an important role seems to carry over to the spinning case, so we focussed on the effect of the Hamiltonian. Starting evolutions at separations between $D=10 \ldots 12 M$ we varied the spin magnitude. Table $\mathrm{V}$ gives the corresponding initial parameters and also shows the effect of constraint solving. For spinning holes the bare masses $\tilde{m}_{1}$ and $\tilde{m}_{2}$ are reduced to keep the total energy constant. In our notation $m_{a}$ replaces the capital $M_{a}$ often used in the numerical literature in order to maintain the PN notation. 
a)

\begin{tabular}{ccc}
\hline \hline$\chi$ & \multicolumn{2}{c}{$e_{D}$ (PN evolution) } \\
& $D=10 M$ & $D=8 M$ \\
\hline-0.25 & 0.0020 & 0.0040 \\
0.00 & $1 \cdot 10^{-7}$ & $3 \cdot 10^{-7}$ \\
0.25 & 0.0015 & 0.0024 \\
0.50 & 0.0050 & 0.0083 \\
0.85 & 0.014 & 0.022 \\
\hline
\end{tabular}

b)

\begin{tabular}{rll}
\hline \hline \multicolumn{1}{c}{$\chi$} & $e_{D}(\mathrm{TH})$ & \multicolumn{1}{c}{$e_{D}(\mathrm{EH})$} \\
\hline-0.25 & $0.015 \pm 0.002$ & $0.005 \pm 0.001$ \\
0.00 & $0.002 \pm 0.001$ & $0.003 \pm 0.001$ \\
0.25 & $0.015 \pm 0.003$ & $0.0015 \pm 0.0005$ \\
0.50 & $0.034 \pm 0.005$ & $0.005 \pm 0.001$ \\
0.85 & $0.07 \pm 0.01$ & $0.014 \pm 0.003$ \\
\hline \hline
\end{tabular}

TABLE IV: a) Eccentricities from conservative PN evolution for aligned equal spins at $D=10 M$ and $D=8 M$. b) Results from corresponding simulations with BAM at comparable binary separations, cf. Fig. 7.

\begin{tabular}{cccccccccc}
\hline \hline \multirow{2}{*}{ Mass ratio } & $\chi$ & $D / M$ & Data type & $P_{t} / M$ & $P_{R} / 10^{-3} M$ & $m_{1}$ & $m_{2}$ & $\tilde{m}_{1}$ & $\tilde{m}_{2}$ \\
\hline \multirow{2}{*}{$1: 1$} & -0.25 & \multirow{2}{*}{12} & THTF & 0.086803 & -0.60477 & 0.5 & 0.5 & 0.4757 & 0.4757 \\
& & & EHPF & 0.086459 & -0.57913 & 0.5 & 0.5 & 0.4757 & 0.4757 \\
\hline $1: 1$ & 0.00 & \multirow{2}{*}{11} & THTF & 0.090110 & -0.71442 & 0.5 & 0.5 & 0.4872 & 0.4872 \\
& & & EHPF & 0.090266 & -0.71489 & 0.5 & 0.5 & 0.4872 & 0.4872 \\
\hline $1: 1$ & 0.25 & \multirow{2}{*}{12} & THPF & 0.083322 & -0.47391 & 0.5 & 0.5 & 0.4758 & 0.4758 \\
& & & EHPF & 0.083858 & -0.49460 & 0.5 & 0.5 & 0.4758 & 0.4758 \\
\hline $1: 1$ & \multirow{2}{*}{0.50} & \multirow{2}{*}{11} & THPF & 0.085921 & -0.54782 & 0.5 & 0.5 & 0.4328 & 0.4328 \\
& & & EHPF & 0.087194 & -0.60170 & 0.5 & 0.5 & 0.4328 & 0.4328 \\
\hline $1: 1$ & 0.85 & \multirow{2}{*}{10} & THPF & 0.087364 & -0.60110 & 0.5 & 0.5 & 0.2564 & 0.2564 \\
& & & EHPF & 0.090080 & -0.71349 & 0.5 & 0.5 & 0.2563 & 0.2563 \\
\hline $4: 1$ & 0.00 & \multirow{2}{*}{10} & THTF & 0.061921 & -0.43393 & 2.0 & 0.5 & 1.9788 & 0.4761 \\
& & & EHPF & 0.061883 & -0.42106 & 2.0 & 0.5 & 1.9788 & 0.4761 \\
\hline \hline
\end{tabular}

TABLE V: Initial parameters used in the simulations with spin for equal masses. The same quantities are shown for a 4:1 run for comparison. The total mass is $M=m_{1}+m_{2}$.

Fig. 7 depicts the resulting eccentricity graphs while Table IV]b provides the corresponding numbers. Fig. 8 shows additional details for the data in Fig. 7, including results obtained by other methods.

The main observation is that the eccentricity increases significantly as $\chi$ is changed from zero towards positive and negative values. For TH data, the minimum is near $\chi=0$, while for $\mathrm{EH}$ data the minimum is closer to $\chi=0.25$ than $\chi=0$. The EOB data yield remarkably low eccentricities, and for $\chi \geq 0.25$ the values are very close to the PN-limit estimate of Table IV]a. The TH data perform poorly in comparison, with up to five times larger eccentricity for $|\chi| \geq 0.25$. It would be interesting to extend this study to more negative values of $\chi$. In order to illustrate these results Fig. 9 shows the black-hole orbits of the simulation with $\chi=0.85$. Strong deviations from a spiral can be observed for the Taylor-Hamiltonian data while the EOB data produce a rather well-formed inspiral. Note that the motion takes place in the $z=0$ plane.

As a first step towards exploring more general spin configurations, we consider one particular example with arbitrarily chosen spin vectors in Fig. $7 \mathrm{f}$. In this case the motion does not remain in the $z=0$ plane and the orbital plane shows considerable precession, see Fig. 10 . Both spins are of magnitude $\chi=0.5$ with a smaller but positive component in the $z$-direction, and the eccentricity is indeed in the range that we would expect from the graphs Fig. 7 c-d where spins of magnitude 0.25 and 0.5 are aligned to the $z$-direction. This indicates that our initial data are not restricted to aligned spins only, but also lead to small eccentricity for more general spins.

Finally, let us look at the spin-spin interaction issue once more. As stated in the discussion of the aligned spin setup, the spin-spin interaction term simplifies in this case and might be incorporated in the initial data computation. This was done in Fig. 8 and indeed eccentricity is reduced by a factor two for the EH data. This approach, however, cannot amend the poor performance of the TH initial parameters.

After noting the effect of the different initial data types, let us investigate how the large differences can be explained by having a closer look at the particular values of $P_{t}$ and $P_{R}$. In the equal-mass setup without spins the differences among the initial parameters were comparatively small even up to close binary separations, Figs. 2 and 3 . When incorporating spins (parallel to $\boldsymbol{L}$ ) we observe the modifications to tangential and radial momentum shown in Fig. 11. As a first finding the tangential component experiences significant changes all over the spin-parameter space. This can be explained by means of frame dragging. Spacetime is twisted in the vicinity 
a)

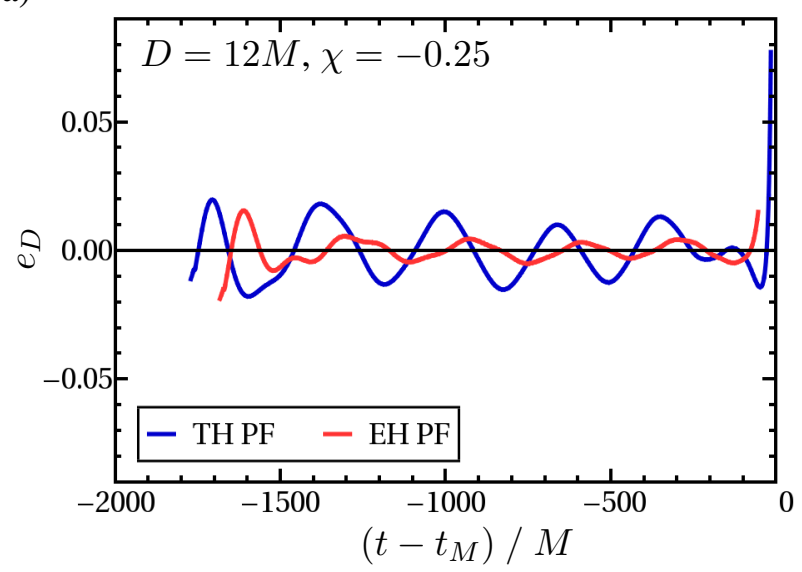

c)

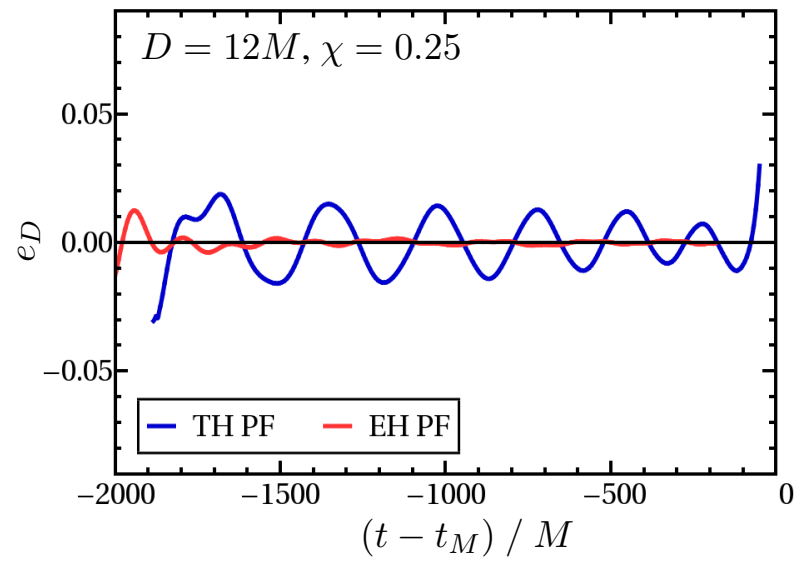

e)

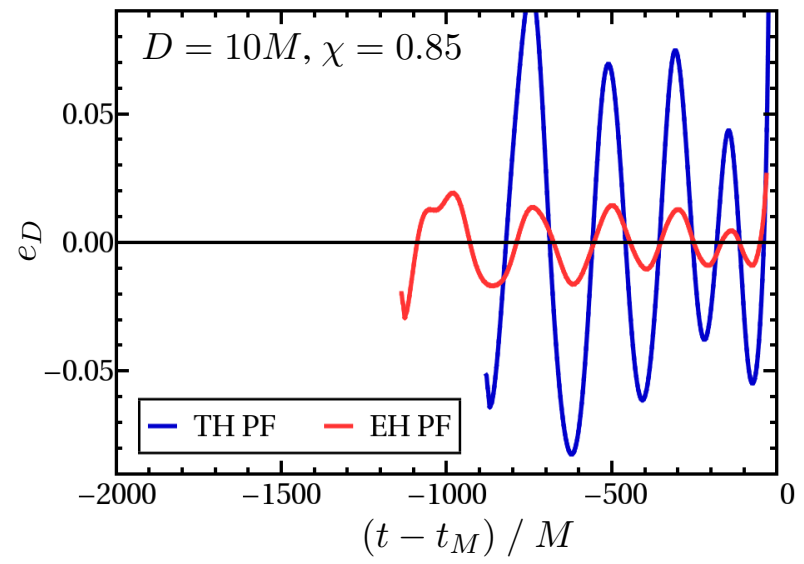

b)

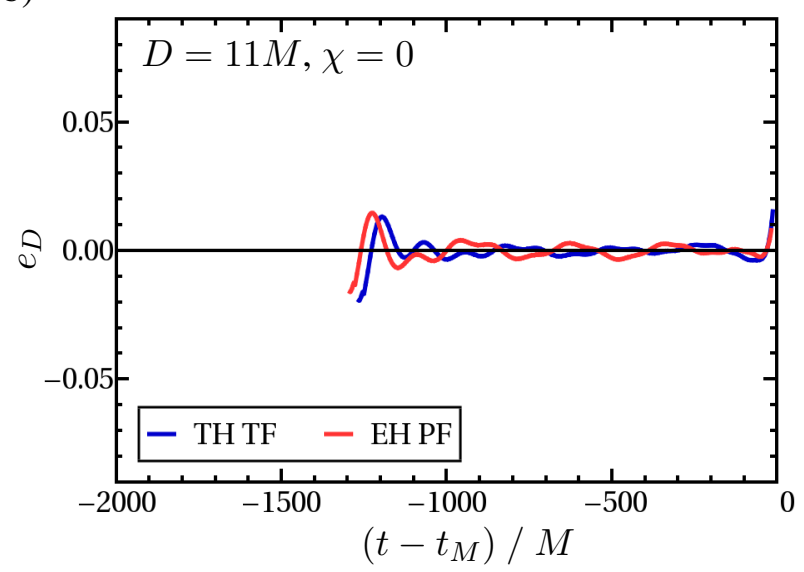

d)

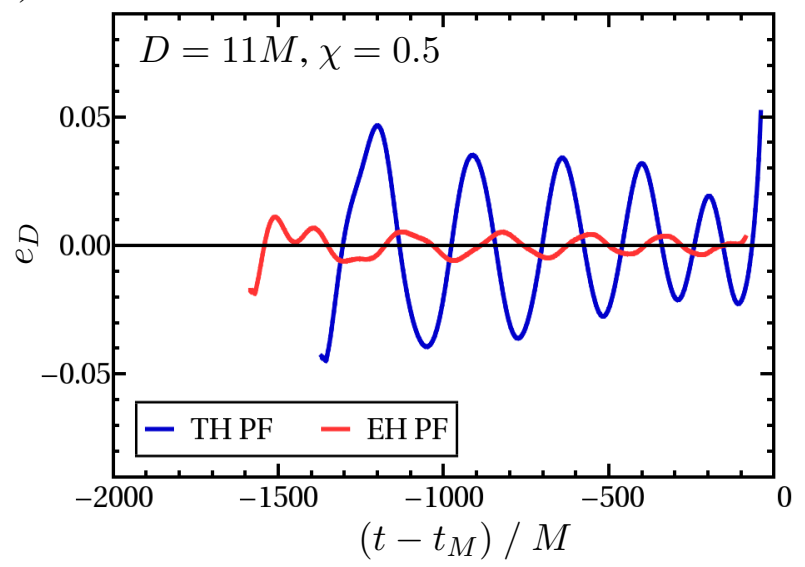

f)

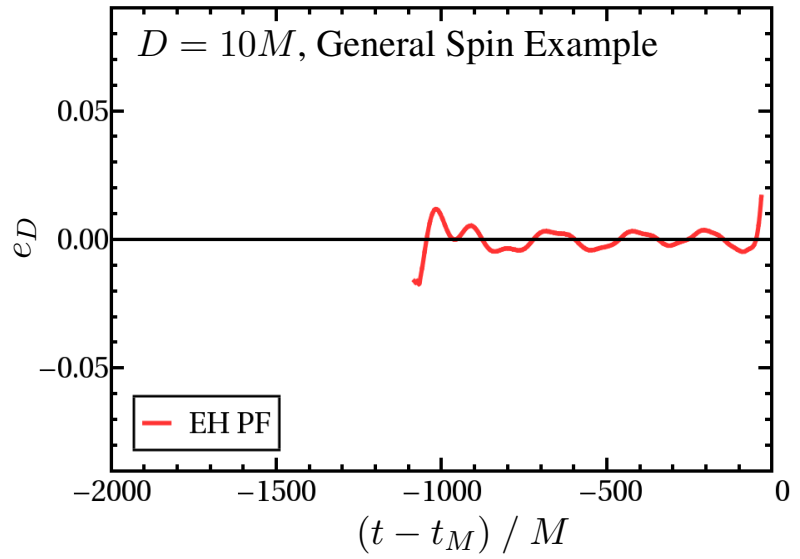

FIG. 7: (Color online) Performance of PN initial data with spin in numerical simulations. In the present equal-mass configurations $\left(m_{1}=m_{2}=0.5\right)$, both spin vectors point in the same direction parallel to the orbital angular momentum and have the same length, i.e. $\boldsymbol{S}_{i}=G m_{i} / c^{2} \cdot \chi \hat{\boldsymbol{L}}$ for panels (a) - (e). We compare the eccentricity $e_{D}$ between TH and EH data for various values of $\chi$. In panel (f) we set up a more general spin configuration with $\boldsymbol{S}_{1}=m_{1}^{2} \chi_{1}\left(0,-\cos 45^{\circ}, \cos 45^{\circ}\right)$ and $\boldsymbol{S}_{2}=m_{2}^{2} \chi_{2}\left(-\cos 60^{\circ}, 0, \cos 30^{\circ}\right)$ with $\chi_{1}=\chi_{2}=0.5$. In order to simplify comparisons of the plots, all axes are scaled the same way, and $t_{M}$ denotes the moment of merger. Large amplitudes in the beginning of the graphs are due to gauge adjustments in the code and should be ignored, while strong drifts near the end of some curves arise when the fit function (needed to compute $\left.e_{D}\right)$ becomes inappropriate. 
a)

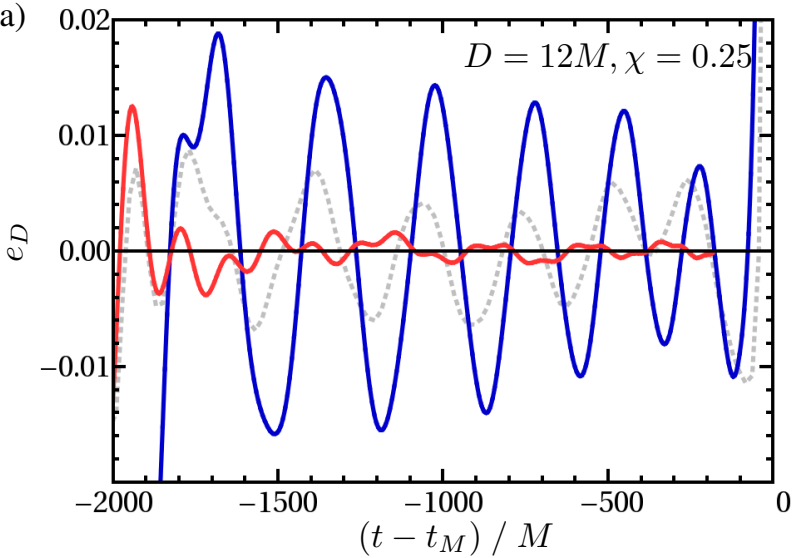

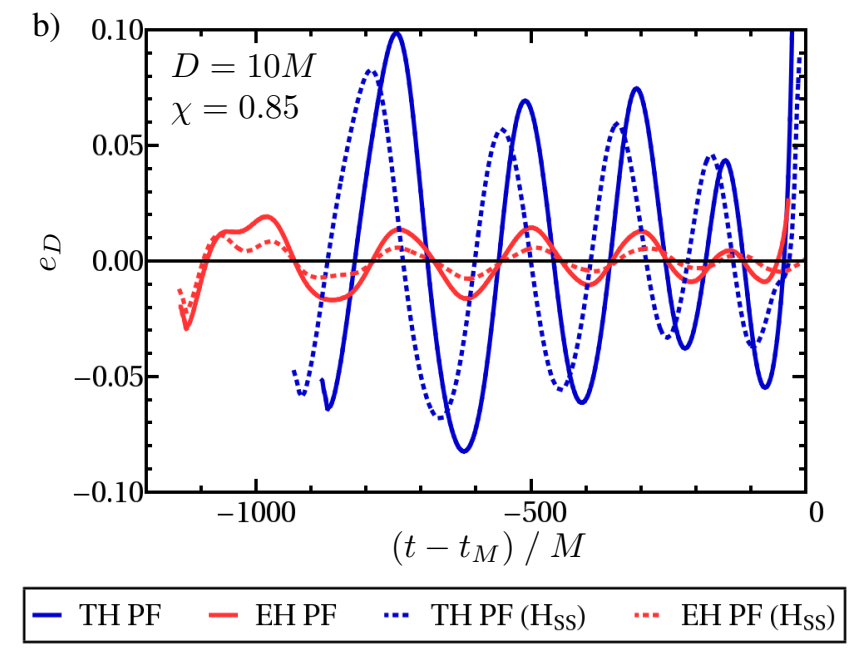

TH PF

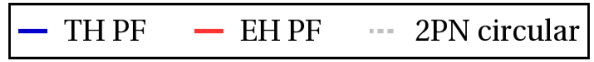

FIG. 8: (Color online) Zoom-in plots for some of the cases of Fig. 7 comparing to eccentricities obtained with other methods. a) For equal masses and spin $\chi=0.25,2 \mathrm{PN}$-circular data $\left(P_{R}=0\right)$ amended with spin (see [56]) happen to give larger eccentricity than the EH data with properly chosen $P_{R}$. b) For equal masses and spin $\chi=0.85$, the benefits of including spin-spin interactions in the initial data computation are shown.

of each spinning hole and the other object is forced to follow this twist. When both spins are aligned with the orbital angular momentum each black hole tries to accelerate the other on its orbit, with the effect that less orbital momentum $P_{t}$ is needed for a circular inspiral. (Recall that it is the velocity which is responsible for the orbit's shape and that there is a complicated, spindependent relation between $\dot{\boldsymbol{X}}=\partial H\left(\boldsymbol{X}, \boldsymbol{P}, \boldsymbol{S}_{1}, \boldsymbol{S}_{2}\right) / \partial \boldsymbol{P}$ and the momentum.)

The TH and EH surfaces almost coincide in the nonspinning case, which marks the center of the plots. With increasing spins the Taylor-expanded data exhibit a stronger reaction than the EOB ones. Recall that comparatively small changes in $P_{t}$ can have strong effects on the orbit. The TH and EH surfaces for $P_{R}$ are almost identical for a certain part of the parameter space, in particular as long as both spins are positive. For strong negative spins, especially for the dominant spin of the bigger black hole at mass ratio 4:1, large changes in $P_{R}$ occur. Obviously, the Taylor-expanded data are affected most, resulting in a behavior similar to Fig. $4 \mathrm{~d}$, such that no reasonable solution is obtained beyond a certain spin threshold. Considering their poor performance in numerical runs, we conclude that TH data are as inappropriate in this regime as in the non-spinning case at high mass ratios.

The EOB-based data do surprisingly well recalling the difficulties that arise from the transformation EOB $\rightarrow$ ADM. The systematic simplifications we imposed, in particular the approximation concerning the binary separation $R^{\prime}$ entering the spin-orbit Hamiltonian, Eq. (16) resp. 40, do not seem to significantly degrade their performance.

\section{CONCLUSIONS}

Based on the work of 28 we presented several variations of an (almost) analytical algorithm capable of delivering quasi-spherical initial data for black-hole binary systems. By employing two different PN Hamiltonians in combination with two versions of energy-flux functions we constructed four types of initial parameters. For the resulting initial data we performed numerical simulations within the moving puncture framework with the BAM code, both for non-spinning and spinning binaries, where we focussed on the class of aligned spins. An eccentricity measurement provided information on the quality of the data. In summary, the eccentricity of the initial data considered grows for smaller binary separations, larger mass ratios and larger spin magnitudes.

In the non-spinning case, differences in the initial parameters $P_{t}$ and $P_{R}$ as well as in the resulting eccentricities were found to be comparatively small and often beyond the accuracy of our measurement. However, TH data happen to suffer from a parasitic pole at higher mass ratios. In particular due to the $\mathrm{EH}$ data we were able to meet or even to obtain slightly lower eccentricities than those resulting from the PN-evolution method of [21. As in 21, a non-zero radial component of the initial momentum is required for best results.

As soon as spins are incorporated, PN theory shows that non-eccentric inspirals are not possible in general due to spin-spin interactions, but we demonstrated that EH initial data are suitable to minimize the radial oscillations to a certain degree, at least for special, aligned spin constellations. We considered one example for nonaligned spins, for which a similarly small eccentricity was obtained. Further runs with arbitrary spin directions are 

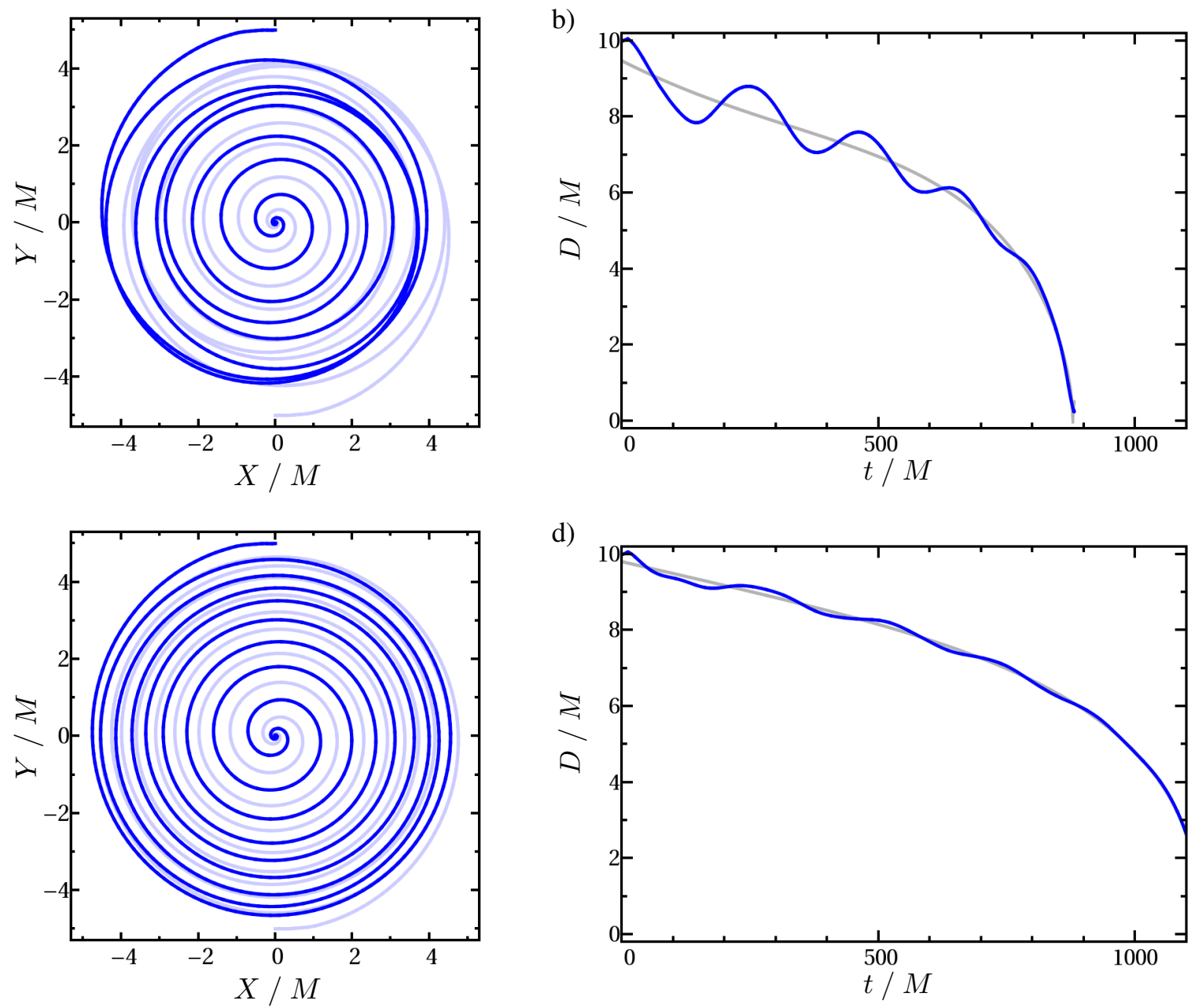

FIG. 9: (Color online) Puncture tracks (left panels) and puncture distance (right panels) for the $\chi=0.85$ simulation from Fig. 7 e. The upper plots pertain to the Taylor-expanded data (THPF) and give a large eccentricity of $e_{D}=0.07$, whereas the EOB data (EHPF) yield much smaller fluctuations in the binary separation leading to $e_{D}=0.014$ (lower plots). The right-hand-side graphs also indicate the procedure of eccentricity measurement. The grey/lighter curve is the fit function $D_{c}(t)$ representing an ideal, non-eccentric inspiral.

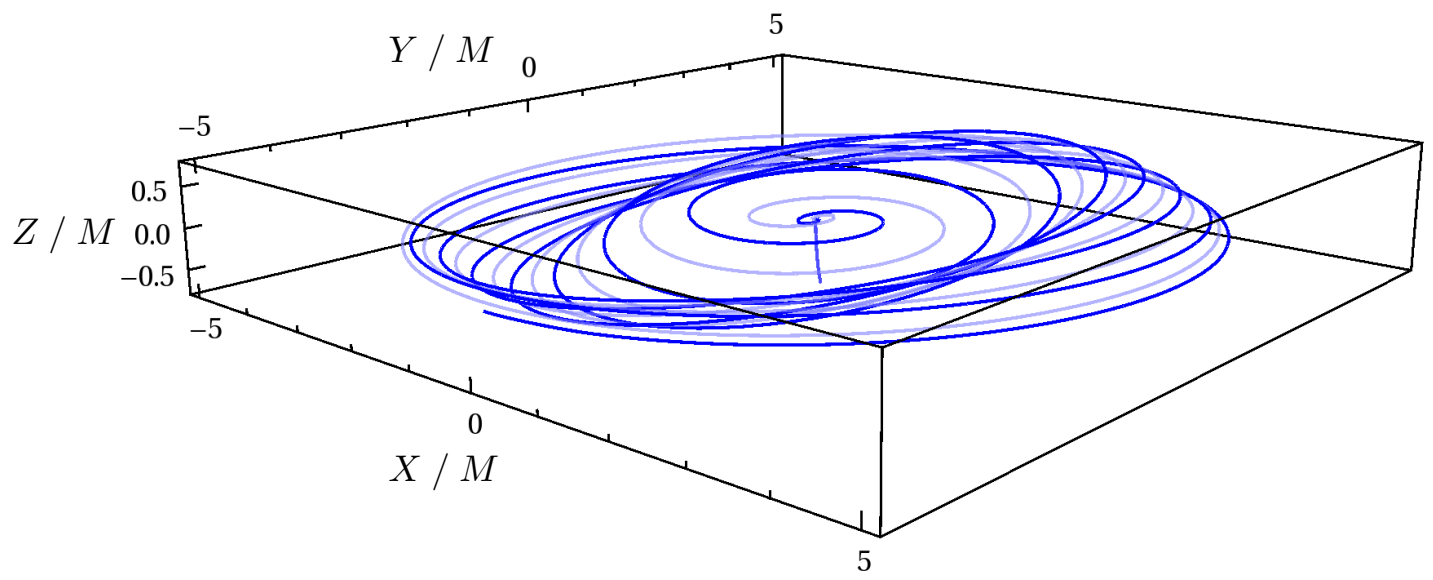

FIG. 10: (Color online) Orbital motion for the general spin example from Fig. 7 f. The orbital plane precesses and we observe a spin kick of the final black hole. 
a)

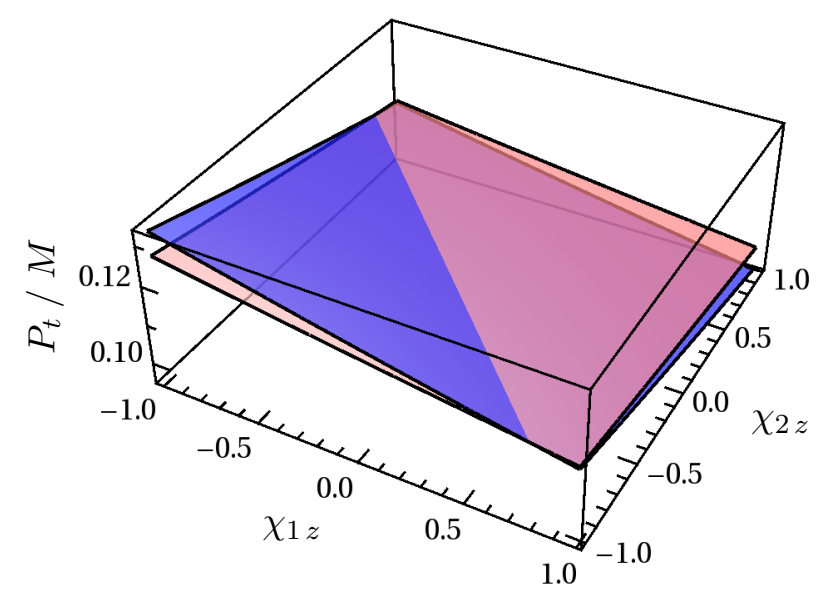

c)

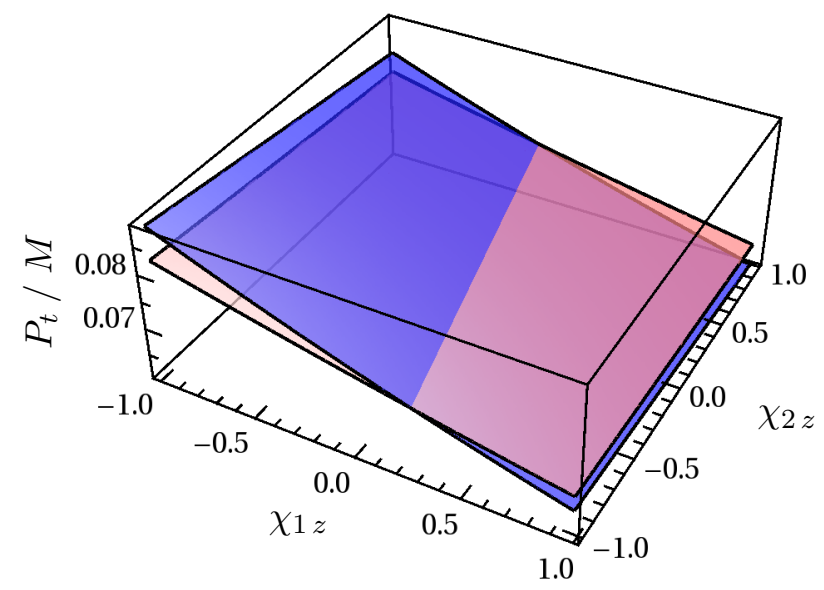

b)

mass ratio $1: 1$

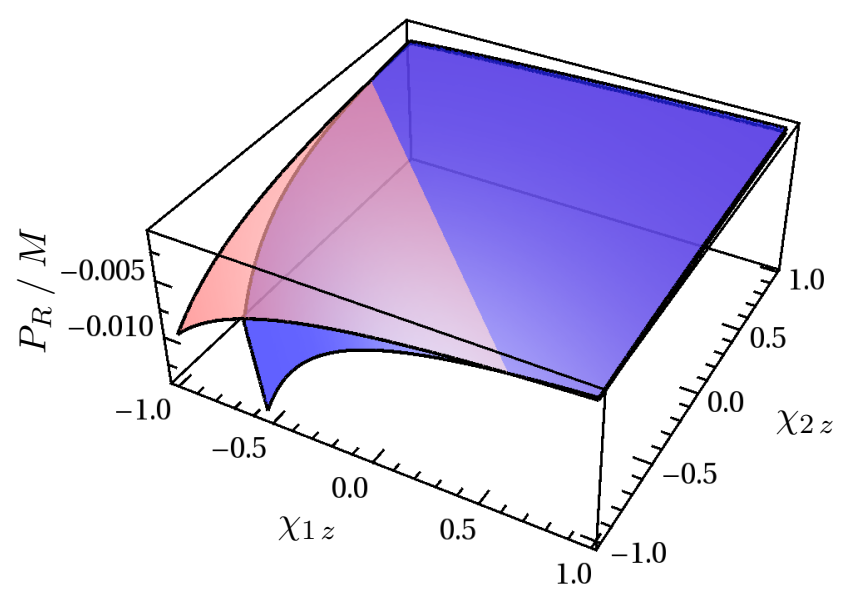

d)

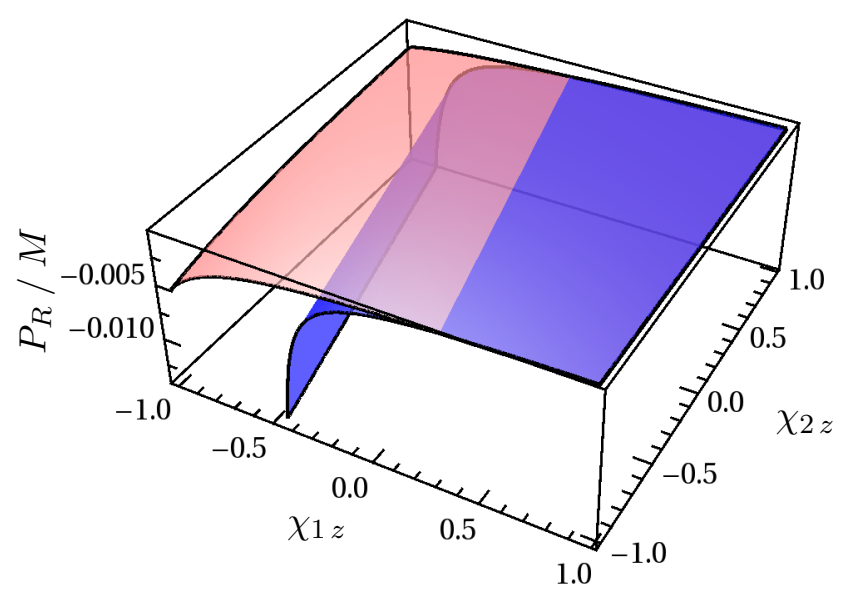

FIG. 11: (Color online) Modifications of PN initial parameters $P_{t}$ and $P_{R}$ for a binary at a fixed initial separation of $8 M$ when spins are involved. The plots show THTF (blue/dark) and EHTF (red/light) initial data surfaces, for which the $z$ component of the spins is varied in the interval $[-1,+1]$. The zero-spin case marks the center of the plots, while e.g. the $\chi_{1 z}=\chi_{2 z}=1$ case corresponds to maximally-spinning black holes with spins aligned to the orbital angular momentum. Changing to Padé-resummed flux does not cause visible modifications of the surfaces.

planned for future research. As expected, the eccentricities in simulations with spins turned out to be larger than the spinless ones. The TH data gave comparatively poor results, which again can be considered an effect of the pole mentioned above.

To end with a concrete suggestion for practical implementations, a good starting point is the EOB Hamiltonian with the Taylor expanded flux, especially for nonzero spin, but also for mass ratios 4:1 and larger. For smaller mass ratios the Taylor Hamiltonian gives smaller eccentricities, but the eccentricity for both $\mathrm{EH}$ and $\mathrm{TH}$ is quite small. The main complication in the EOB method is the need for the transformation to ADMTT coordinates for the numerical initial data. Table III gives explicit values for initial parameters for several nonspinning binary configurations that can be used directly in simulations. Use the combination of $P_{t}$ and $P_{R}$ for which the eccentricity given in the last column of the table is lowest. Initial data for several binary configurations with (anti-)aligned spins can be read off table $\mathrm{V}$. For all cases with $\chi \neq 0$, the EHPF data leads to the lowest eccentricity.

Further investigations suggest themselves. The PNevolution method of 21] depends on the quality and char- 
acteristics of the Hamiltonian evolution method. It is natural to revisit this topic with the insights gained in the present work for unequal masses and spins, and also to compare with the PN-evolution methods of 26, 27]. Our study centers on the PN method of [28] and numerical evolutions. A refined method called "post-post-circular" initial data has been suggested in [57, which is worth investigating in the context of numerical evolutions as well.

Apart from evolutions, it could be interesting to compare to other diagnostics for the eccentricity of orbits, e.g. [12, [58, 59].

An alternative to the TH and EH methods considered here is the semi-analytic puncture evolution approach to model inspiralling black holes 60. It is natural to expect that the resulting orbital parameters are well adapted to numerical puncture evolutions, and we plan to carry out a quantitative study.

\section{Acknowledgments}

It is a pleasure to thank Achamveedu Gopakumar, Mark Hannam, Sascha Husa, and Gerhard Schäfer for discussions and valuable insights in the PN method. This work was supported in part by DFG grant SFB/Transregio 7 "Gravitational Wave Astronomy" and the DLR (Deutsches Zentrum für Luft und Raumfahrt). Computations were performed on the HLRB2 at LRZ Munich.
[1] H. Lück et al., Class. Quantum Grav. 23, S71 (2006).

[2] B. Abbott and the LIGO Scientific Collaboration (2007), arXiv:0711.3041 [gr-qc].

[3] D. Tatsumi et al., Class. Quant. Grav. 24, S399 (2007), arXiv:0704.2881v1 [gr-qc].

[4] F. Acernese et al., Class. Quant. Grav. 24, S381 (2007).

[5] F. Pretorius (2007), arXiv:0710.1338v1 [gr-qc].

[6] L. Blanchet, Living Rev. Relativity 5, 3 (2002), gr-qc/0202016, URL http://www.livingreviews.org/ lrr-2002-3.

[7] B. Aylott, J. G. Baker, W. D. Boggs, M. Boyle, P. R. Brady, D. A. Brown, B. Brügmann, L. T. Buchman, A. Buonanno, L. Cadonati, et al., Class. Quant. Grav. 26, 165008 (2009), arXiv: 0901.4399 [gr-qc].

[8] G. B. Cook, Living Rev. Relativity 3, 5 (2000), URL http://www . livingreviews.org//rr-2000-5.

[9] H. P. Pfeiffer, D. A. Brown, L. E. Kidder, L. Lindblom, G. Lovelace, and M. Scheel, Class. Quant. Grav. 24, S59 (2007), gr-qc/0702106.

[10] B. Brügmann, J. A. González, M. Hannam, S. Husa, U. Sperhake, and W. Tichy, Phys. Rev. D77, 024027 (2008), gr-qc/0610128.

[11] J. Baker, M. Campanelli, C. O. Lousto, and R. Takahashi, Phys. Rev. D 65, 124012 (2002), astroph/0202469.

[12] E. Berti, S. Iyer, and C. M. Will, Phys. Rev. D 74, 061503 (2006), gr-qc/0607047.

[13] S. Brandt and B. Brügmann, Phys. Rev. Lett. 78, 3606 (1997), gr-qc/9703066.

[14] A. Buonanno and T. Damour, Phys. Rev. D 59, 084006 (1999), gr-qc/9811091.

[15] A. Buonanno and T. Damour, Phys. Rev. D 62, 064015 (2000), gr-qc/0001013.

[16] T. Damour, P. Jaranowski, and G. Schäfer, Phys. Rev. D 62, 084011 (2000), gr-qc/0005034.

[17] T. Damour, Phys. Rev. D 64, 124013 (2001), grqc/0103018.

[18] W. Tichy, B. Brügmann, M. Campanelli, and P. Diener, Phys. Rev. D 67, 064008 (2003), gr-qc/0207011.

[19] B. J. Kelly, W. Tichy, M. Campanelli, and B. F. Whiting, Phys. Rev. D76, 024008 (2007), arXiv:0704.0628v2 [grqc].
[20] N. Hernandez and R. H. Price (2008), 0812.4443.

[21] S. Husa, M. Hannam, J. A. González, U. Sperhake, and B. Brügmann, Phys. Rev. D77, 044037 (2008), arXiv:0706.0904v1 [gr-qc].

[22] L. E. Kidder, Phys. Rev. D 52, 821 (1995), grqc/9506022.

[23] B. Brügmann, J. A. González, M. Hannam, S. Husa, and U. Sperhake, Phys. Rev. D77, 124047 (2008), arXiv:0707.0135v1 [gr-qc].

[24] M. Hannam, S. Husa, J. G. Baker, M. Boyle, B. Brügmann, T. Chu, N. Dorband, F. Herrmann, I. Hinder, B. J. Kelly, et al., Phys. Rev. D 79, 084025 (2009), arXiv:0901.2437 [gr-qc].

[25] T. Damour, A. Nagar, M. Hannam, S. Husa, and B. Brügmann, Phys. Rev. D78, 044039 (2008), arXiv:0803.3162v1 [gr-qc].

[26] M. Campanelli, C. O. Lousto, H. Nakano, and Y. Zlochower (2008), arXiv:0808.0713v2 [gr-qc].

[27] S. Husa and M. Hannam, Presentations at Post-Newton 2008 (2008), URL http://wwwsfb.tpi.uni-jena.de/ Events/PN2008/PN2008_Program.shtml.

[28] A. Buonanno, Y. Chen, and T. Damour, Phys. Rev. D74, 104005 (2006), gr-qc/0508067.

[29] T. Damour, P. Jaranowski, and G. Schäfer, Phys. Rev. D 62, 044024 (2000), gr-qc/9912092.

[30] T. Damour, P. Jaranowski, and G. Schäfer, Phys. Rev. D62, 021501 (2000), erratum-ibid. 63, 029903, (2000), gr-qc/0003051.

[31] T. Damour, P. Jaranowski, and G. Schäfer, Phys. Lett. B 513, 147 (2001), gr-qc/0105038.

[32] T. Damour and G. Schäfer, Nuovo Cimento B 101, 127 (1988).

[33] L. Blanchet and G. Schäfer, Monthly Notices of the Royal Astronomical Society 239, 845 (1989).

[34] W. Junker and G. Schäfer, Monthly Notices of the Royal Astronomical Society 254, 146 (1992).

[35] L. Blanchet, G. Faye, B. R. Iyer, and B. Joguet, Phys. Rev. D65, 061501 (2002), gr-qc/0105099.

[36] L. Blanchet, G. Faye, B. R. Iyer, and B. Joguet, Phys. Rev. D 71, 129902 (2005).

[37] L. Blanchet, T. Damour, G. Esposito-Farese, and B. R. Iyer, Phys. Rev. Lett. 93, 091101 (2004), gr-qc/0406012. 
[38] K. G. Arun, L. Blanchet, B. R. Iyer, and M. S. S. Qusailah, Physical Review D 77, 064035 (2008), arXiv: 0711.0302 [gr-qc].

[39] A. H. Mroué, L. E. Kidder, and S. A. Teukolsky, Phys. Rev. D78, 044004 (2008), arXiv:0805.2390v3 [gr-qc].

[40] T. Damour, B. R. Iyer, and B. S. Sathyaprakash, Phys. Rev. D 57, 885 (1998), gr-qc/9708034.

[41] E. K. Porter and B. S. Sathyaprakash, Physical Review D 71, 024017 (2005).

[42] T. Damour, P. Jaranowski, and G. Schäfer (2008), arXiv:0803.0915v1 [gr-qc].

[43] A. Buonanno, G. B. Cook, and F. Pretorius, Phys. Rev. D75, 124018 (2007), gr-qc/0610122.

[44] M. Boyle et al., Phys. Rev. D76, 124038 (2007), arXiv:0710.0158v2 [gr-qc].

[45] J. G. Baker, J. R. van Meter, S. T. McWilliams, J. Centrella, and B. J. Kelly, Phys. Rev. Lett. 99, 181101 (2007), gr-qc/0612024.

[46] B. Brügmann, W. Tichy, and N. Jansen, Phys. Rev. Lett. 92, 211101 (2004), gr-qc/0312112.

[47] M. Shibata and T. Nakamura, Phys. Rev. D 52, 5428 (1995).

[48] T. W. Baumgarte and S. L. Shapiro, Phys. Rev. D 59, 024007 (1998), gr-qc/9810065.

[49] M. Campanelli, C. O. Lousto, P. Marronetti, and Y. Zlochower, Phys. Rev. Lett. 96, 111101 (2006), grqc/0511048.
[50] J. G. Baker, J. Centrella, D.-I. Choi, M. Koppitz, and J. van Meter, Phys. Rev. Lett. 96, 111102 (2006), grqc/0511103.

[51] S. Husa, J. A. González, M. Hannam, B. Brügmann, and U. Sperhake, Class. Quantum Grav. 25, 105006 (2008), arXiv:0706.0740 [gr-qc].

[52] C. Bona, J. Massó, E. Seidel, and J. Stela, Phys. Rev. Lett. 75, 600 (1995), gr-qc/9412071.

[53] M. Alcubierre, B. Brügmann, P. Diener, M. Koppitz, D. Pollney, E. Seidel, and R. Takahashi, Phys. Rev. D 67, 084023 (2003), gr-qc/0206072.

[54] C. Gundlach and J. M. Martin-Garcia, Phys. Rev. D 74, 024016 (2006), gr-qc/0604035.

[55] J. A. González, U. Sperhake, and B. Brügmann, Phys. Rev. D79, 124006 (2009), arXiv: 0811.3952 [gr-qc].

[56] M. Hannam, S. Husa, B. Brügmann, and A. Gopakumar, Phys. Rev. D78, 104007 (2008), arXiv:0712.3787 [gr-qc].

[57] T. Damour, A. Nagar, E. N. Dorband, D. Pollney, and L. Rezzolla, Phys. Rev. D77, 084017 (2008), 0712.3003.

[58] T. Mora and C. M. Will, Phys. Rev. D69, 104021 (2004), gr-qc/0312082.

[59] J. D. Grigsby and G. B. Cook, Phys. Rev. D77, 044011 (2008), arXiv:0706.4286 [gr-qc].

[60] A. Gopakumar and G. Schäfer, Phys. Rev. D77, 104023 (2008), arXiv:0803.2348 [gr-qc]. 